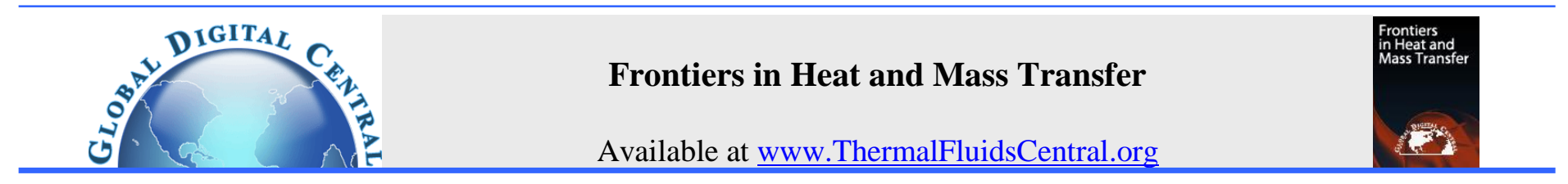

\title{
HEAT AND MASS TRANSFER ANALYSIS ON MHD MIXED CONVECTION FLOW OF RADIATIVE CHEMICALLY HEAT GENERATING FLUID WITH VISCOUS DISSIPATION AND THERMO-DIFFUSION EFFECT
}

\author{
Sanjib Sengupta ${ }^{\mathrm{a}, *}$ Amrit Karmakar ${ }^{\mathrm{b}}$
}

\author{
${ }^{a}$ Department of Mathematics, Assam University, Silchar, Assam, 788011, India \\ bJawahar Navodaya Vidyalaya, R. C. Ghat, Khowai, Tripura, 799207, India
}

\begin{abstract}
In this paper an analysis on heat and mass transfer is made to study magnetohydrodynamic (MHD) mixed convective flow of an incompressible viscous fluid flowing past an inclined plate. A magnetic field of uniform strength is applied to the plate to influence the flow. Due to weak voltage differences caused by the very low polarization charges, the influence of electric field is considered to be neglected. Again large temperature gradient ensures cross diffusion effect like thermo-diffusion (Soret) in the field. The governed set of non-linear partial differential equations is solved by developing a multi-parameter asymptotic perturbation scheme. The influence of various physical parameters such as heat source parameters $(Q s)$, chemical reaction parameter $\left(C_{R}\right)$, magnetic field parameter $(M)$, Eckert number $(E c)$, thermal radiation parameter $(R)$, permeability parameter $(K)$ and plate inclination parameter $(\psi)$ on the velocity, concentration and temperature profiles as well as skin-fraction, Nusselt number and Sherwood number are simulated numerically for the study. It reveals that, an increase in magnetic field parameter $(M)$ decreases the axial velocity field, coefficient of skin-friction and Sherwood number but increases Nusselt number, temperature and concentration profiles. Again an increase in Eckert number $(E c)$ decreases both the co-efficient of skin-friction and Nusselt number while increases the temperature, Sherwood number and concentrations of the fluid particles.
\end{abstract}

Keywords: MHD mixed convection, porous medium, heat source, chemical reaction, thermal radiation, viscous dissipation, Thermo-diffusion.

\section{INTRODUCTION}

Magneto-hydrodynamic (MHD) is the study of an electromagnetic field interacting with the velocity field of an electrically conducting fluid. It played a vital role in the field of astrophysics, geophysics, nuclear reactor and many electronics devices. Manglesh and Gorla (2012) studied the effects of thermal radiation, chemical reaction and rotation on unsteady MHD visco-elastic slip flow. Ahmed etal (2013) made an exact analysis for MHD free convection mass transfer flow past an oscillating plate embedded in a porous medium with Soret effect. Nasir Uddin et al. (2014) considered the effect of conjugate heat and mass transfer on MHD mixed convective flow past inclined porous plate in porous medium. Heat and mass transfer are kinetic process that may take place jointly or separately, it can be study combined or separately. Heat and mass transfer has a significant role in many industrials and environmental processes particularly in energy utilization, thermal processing, in aerospace, thermal control and food processing. Rajuet al. (2015) analyzedheat and mass transfer in MHD mixed convection flow on a movinginclined porous plate. Sengupta (2015) investigated free convective chemically absorption fluid past an impulsively accelerated plate with thermal radiation variable wall temperature and concentrations. Sengupta and Karmakar (2016) studied the MHD mixed convection chemically reactive flow in radiative heat generating medium with Soret effect. Prashant et al. (2016) investigated heat transfer in MHD mixed convection viscoelastic fluid flow over a stretching sheet embedded in a porous medium with viscous dissipation and non-uniform heat source/sink. Misra and Adhikary (2016) studied the MHD oscillatory channel flow, heat and masstransfer in a physiological fluid in presence ofchemical reaction. Mondal et al (2017) investigated thermophoresis and soret-dufour on MHD mixed convection mass transfer over an inclined plate with non-uniform heat source/sink and chemical reaction.
Heat generation (heat source) /absorption (heat sink) which minimizes the longevity of many electronics device, it has significant role in industries as well as in nuclear reactors. So due to importance of heat generation/absorption in industrial and engineering field, many researchers worked on its effects. Ravikumar et al. (2012) studied heat and mass transfer effects on MHD flowof viscous fluid through non-homogeneous porousmedium in presence of temperaturedependent heat source. Bhavanaet al. (2013) investigated the Soret effect on free convective unsteady MHD flow over a vertical plate with heat source.Ramandeviet al. (2017) analyzed combined influence of viscous dissipation and non - uniform heat source/sink on MHD non-Newtonian fluid flow with cattaneo-christov heat flux. Recently Venkataramana et al. (2017) investigated the influence of heat generation (absorption) and thermal radiation on MHD laminar boundary layer flow over a moving cylindrical rod.

Chemical reaction has an important role in chemical reaction process such as polymer production food processing. Due to which researchers draw a kin interest in its effect. Many researchers work on effects of chemical reaction. Saleh et al. (2010) studied heat and mass transfer in MHD visco-elastic fluid flow through a porous medium over a stretching sheet with chemical reaction. Pal and Talukdar (2011) investigated the combined effects of joule heating and chemical reaction on unsteady magnetohydrodynamic mixed convection of a viscous dissipating fluid over a vertical plate in porous media with thermal radiation. Pal and Mondal (2012) studied the influence of chemical reaction and thermal radiation on mixed convection heat and mass transfer over a stretching sheet in Darcian porous medium with Soret and Dufour effects. Ibrahim (2014) considered the effects of chemical reaction on dissipative radiative MHD flow through a porous medium over a non-isothermal stretching sheet. Rudraswamy and Gireesha (2014) studied the influence of chemical reaction and thermal radiation on MHD 
boundary layer flow and heat transfer of a nano fluid over an exponentially stretching sheet. Jonnadula et al. (2015) investigated the influence of thermal radiation and chemical reaction on MHD flow, heat and mass transfer over a stretching surface. Sengupta and Ahmed (2015) studied the effect of chemical reaction interaction on unsteady MHD free convective radiative flow past an oscillating plate embedded in porous media with thermal diffusion. Nayak et al. (2016) studied heat and mass transfer effects on MHD visco-elastic fluid over a stretching sheet through porous medium under the influence of chemical reaction. Jena et al. investigated the chemical reaction effect on MHD viscoelastic fluid flow over a vertical stretching sheet with heat source/sink. Srinivasacharya and Swamy Reddy,(2016) investigated chemical reaction and radiation effects on mixed convection heat and mass transfer over a vertical plate in power-law fluid saturated porous medium.Reddy et al. (2016) studied the influence of chemical reaction, radiation and rotation on MHD nano fluid flow past a permeable flat plate in porous medium.

The process through which the fluids takes energy (kinetic energy) from the motion of the fluid and transform it into internal energy of the fluid is called viscous dissipation. It is partially an irreversible process. Reddyet al. (2015) studied the effects of viscous dissipation and heat source on unsteady MHD flow over a stretching sheet. Of late Ahmed et al. (2017) studied the effects of chemical reaction, heat and mass transfer and viscous dissipation over a MHD flow in a vertical porous wall using perturbation method. Shamshuddin (2017) investigated the influence of heat and mass transfer on the unsteady MHD flow of chemically reacting micropolar fluid with radiation and Joule heating. It is well understood that driving potential for the phenomenon of transport of mass is not only concentration gradients, but also high temperature gradients are also one of the means. The dual process by which mass gets transferred is known as thermo-diffusion or Soret effect. Due to the scientific and industrial importance of Soret effect, many researchers contributed a lot on its effects. Sengupta and Sen (2013) considered the study of thermo-diffusion (Soret) on a free convective heat and mass transfer flow past an oscillating plate with heat generation and thermal radiation. Mamatha et al. (2015) investigated the thermal diffusion effect on MHD mixed convection unsteady flow of a micro polar fluid past a semi-infinite vertical porous plate with radiation and mass transfer. Sengupta (2015) made an analysis of Soret effect on unsteady heat and mass transfer flow of radiative chemically reactive fluid past an oscillating plate embedded in porous media. Rashidi et al. (2015) investigated heat and mass transfer for MHD viscoelastic fluid flow over a vertical stretching sheet with considering Soret and Dufour effects. Reddy et al. (2016) investigated the Soret and Dufour effects on MHD free convection flow of RivlinEricksen fluid past a semi-infinite vertical plate. Sengupta and Ahmed (2016) studied MHD free convective mass transfer flow of radiative uniform heat generation (absorption) fluid through a wavy permeable channel in presence of Soret and Dufour effects. Ullah et al. (2017) investigated Soret as well as Dufour effects on unsteady mixed convection slip flow of Caisson fluid over anonlinearly stretching sheet withconvective boundary condition. Very recently Sengupta and Karmakar (2018) investigated the induced magnetic field interaction in free convective heat and mass transfer flow of a chemically reactive heat generating fluid with thermo-diffusion and diffusion-thermo effects. Shamshuddin et al. (2018) compute the unsteady MHD mixed convective heat and mass transfer in dissipative reactive micropolar flow considering Soret and Dufour effects.

In spite of these studies, less attention has been given by authors to free stream oscillation flow with combine effect of viscous dissipation, thermo-diffusion (Soret) and thermal radiation on a flow of electrically conducting fluid. The objective of the present study is to make an analysis of combine effect of thermo-diffusion (Soret), viscous dissipation and thermal radiation of electrically conducting and chemically reactive fluid in heat generating, Darcian porous media in presence of magnetic field with an inclined plate condition.

\section{BASIC EQUATIONS AND ASSUMPTIONS}

\subsection{Basic Equations}

The vector forms of equations that describe the flow situation are as follows

The velocity vector and the magnetic field vectors are given by

$\vec{q}=(u(y, t), v(y, t), 0)$

The momentum equation gives

$\rho\left(\frac{\partial \vec{q}}{\partial t}+(\vec{q} \cdot \vec{\nabla}) \vec{q}\right)=-\vec{\nabla} p+\mu \vec{\nabla}^{2} \vec{q}+\vec{J} \times \vec{B}-\mu \vec{q}+\rho \vec{g}$

Ohm's law gives,

$\vec{J}=\sigma(\vec{E}+\vec{q} \times \vec{B})$

With electric field $\vec{E}=(0,0,0)$, magnetic field $\vec{B}=\left(0, B_{0}, 0\right)$

and $\sigma$, as the electrical conductivity of the medium.

The vector form of energy equation gives

$\rho C_{p}\left(\frac{\partial T}{\partial t}+(\vec{q} \cdot \vec{\nabla}) T\right)=k \vec{\nabla}^{2} T+Q^{*} \delta T+\Phi$

Where, dissipation function $\Phi=\operatorname{Div}(\tau \vec{v})-\vec{v} \operatorname{Div}(\tau)$

and $\tau$ represents Cauchy's stress tensor.

The species continuity equations as:

$\frac{\partial C}{\partial t}+(\vec{q} \cdot \vec{\nabla}) C=D_{M} \vec{\nabla}^{2} C-K_{l} \delta C+\frac{D_{M} K_{T}}{T_{M}} \vec{\nabla}^{2} T$

The other symbols are specified in the Nomenclature.

\subsection{Basic Assumptions}

The fundamental assumptions considered for the study are as follows: a) All the fluid properties except possibly the pressure are independent of variations of $x *$-scale.

b) The strength of the applied magnetic field, which is applied to the inclined plate, considered as moderate and uniform. Due to the small value of magnetic Reynold's number, the influence of induced magnetic field in comparison with the applied magnetic field is less prominent and thus can be ignored for the study.

c) The Hall as well as magnetic heating effects gets less importance due to moderate strength of the magnetic field.

d) As the applied voltage at the ends of the plate is weak as such polarization of charges is neglected, so the electric field is not considered for the study.

e) Due to higher temperature gradient, the cross diffusion effects, like thermo-diffusion (Soret) have been considered for the study.

f) The plate is considered to be magnetically insulated. The temperature as well as concentration of fluid particles near the plate surface is supposed to be more than their respective components at the free stream region i.e., $\mathrm{T}_{\mathrm{w}}>\mathrm{T}_{\infty}, \mathrm{C}_{\mathrm{w}}>\mathrm{C}_{\infty}$.

\section{MATHEMATICAL FORMULATIONS OF THE PROBLEM AND SOLUTIONS}

A co-ordinate system $\left(x^{*}, y^{*}, z^{*}\right)$ has been introduced, with its $x^{*}$-axis along the length of the plate in the upward vertical direction, $\mathrm{y}^{*}$-axis taken perpendicular to the plate towards the fluid region and $\mathrm{z}^{*}$-axis considered along the width of the plate. A uniform magnetic field of strength $\overrightarrow{\boldsymbol{B}}_{\mathrm{o}}$ is applied to the plate. Following Bhuvaneswari et al. 
(2010) and Barik and Dash (2014), an MHD fluid model has been developed based on conservation principles of mass, momentum, energy and species concentration as well as Boussineque approximations with initio-boundary conditions as:

$$
\frac{\partial v^{*}}{\partial y^{*}}=0
$$

$$
\begin{aligned}
& \frac{\partial u^{*}}{\partial t^{*}}+v^{*} \frac{\partial u^{*}}{\partial y^{*}}=-\frac{1}{\rho} \frac{\partial p^{*}}{\partial x^{*}}+v \frac{\partial^{2} u^{*}}{\partial y^{* 2}}+\left[g \beta_{T}\left(T^{*}-T_{\infty}^{*}\right)+g \beta_{C}\left(C^{*}-C_{\infty}^{*}\right)\right] \cos \psi \\
& -\left(\frac{\sigma B_{0}^{2}}{\rho}+\frac{v}{K^{*}}\right) u^{*}
\end{aligned}
$$

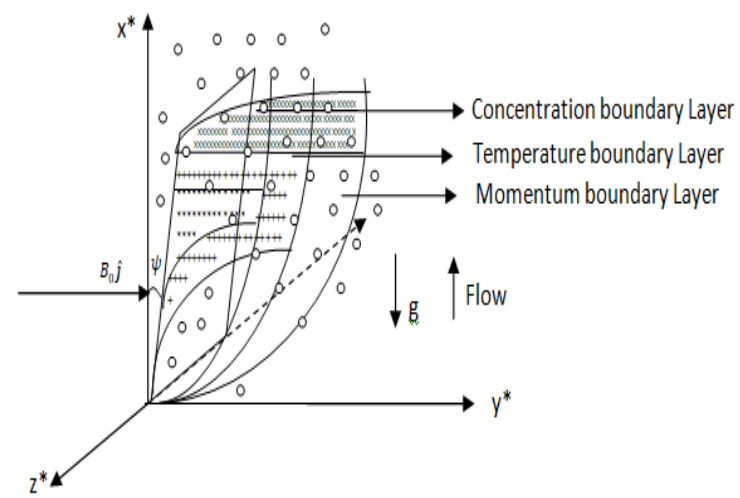

Fig. 1 A Schematic representation of the flow configuration and geometry

$$
\begin{aligned}
& \frac{\partial T^{*}}{\partial t^{*}}+v^{*} \frac{\partial T^{*}}{\partial y^{*}}=\frac{k}{\rho C_{p}} \frac{\partial^{2} T^{*}}{\partial y^{* 2}}-\frac{1}{\rho C_{p}} \frac{\partial q_{r y^{*}}^{*}}{\partial y^{*}}+ \\
& \frac{\mu}{\rho C_{P}}\left(\frac{\partial u^{*}}{\partial y^{*}}\right)^{2}+\frac{Q_{s}^{*}}{\rho C_{p}}\left(T^{*}-T_{\infty}^{*}\right) \\
& \frac{\partial C^{*}}{\partial t^{*}}+v^{*} \frac{\partial C^{*}}{\partial y^{*}}=D_{M} \frac{\partial^{2} C^{*}}{\partial y^{* 2}}+D_{T} \frac{\partial^{2} T^{*}}{\partial y^{* 2}}-K_{l}\left(C^{*}-C_{\infty}^{*}\right)
\end{aligned}
$$

With boundary conditions as:

$u^{*}=0, \mathrm{v}^{*}=0, T^{*}=T_{\infty,}^{*} C^{*}=C_{\infty}^{*}$ for every $y^{*}$ and $t^{*}=0$

$u^{*}=u_{p}^{*}, v^{*}=v_{\omega}^{*}, \frac{\partial T^{*}}{\partial y^{*}}=\frac{-q_{\omega}}{k}$ at $y^{*}=0, t^{*}>0$

$c^{*}=c_{\omega}^{*}+\varepsilon\left(c_{\omega}^{*}-c_{\infty}^{*}\right) \exp \left(i \omega^{*} t^{*}\right)$, at $y^{*}=0$ when $t^{*}>0$

and $u^{*} \rightarrow u_{\infty}^{*}=u_{0}\left(1+\varepsilon \exp \left(i \omega^{*} t^{*}\right)\right), T^{*} \rightarrow T_{\infty}^{*} \& C^{*} \rightarrow C_{\infty}^{*}$ for $y^{*} \rightarrow \infty$, when $t^{*}>0$

Where, $v^{*}=v^{*}{ }_{w}=-\left(1+\varepsilon \lambda \exp \left(i \omega^{*} t^{*}\right)\right)$ is the plate suction velocity.

The hydrostatics pressure in (2) can be calculated from Bernoulli's pressure equations as:

$-\frac{1}{\rho} \frac{\partial p^{*}}{\partial x^{*}}=\frac{\partial u_{\infty}^{*}}{\partial t^{*}}+\left(\frac{\sigma B_{0}^{2}}{\rho}+\frac{v}{K^{*}}\right) u_{\infty}^{*}$

The radiative heat flux for an optically thick boundary layer flow can also be simplified with the Rosseland approximation model as: $q_{r y^{*}}^{*}=\frac{4 \sigma_{1}}{3 k_{1}} \frac{\partial T^{* 4}}{\partial y^{*}}$

Where $\sigma_{1}$ and $k_{1}$ are the Stefan Boltzmann constant and Rosseland mean absorption coefficient respectively. Assuming that the temperature differences with the flow are sufficiently small, so $\mathrm{T}^{* 4}$ may be expressed as:

$$
\begin{aligned}
& F\left(T^{*}\right)=T^{* 4} \\
& F\left(T^{*}\right)=F\left(T_{\infty}^{*}\right)+\left(T^{*}-T_{\infty}^{*}\right) F^{\prime}\left(T_{\infty}^{*}\right)+o\left(T^{*}-T_{\infty}^{*}\right)
\end{aligned}
$$

This gives

$T^{* 4} \approx T_{\infty}^{* 4}+\left(T^{*}-T_{\infty}^{*}\right) 4 T_{\infty}^{* 3}=4 T_{\infty}^{* 3} T^{*}-3 T_{\infty}^{* 4}$

We consider the following dimensionless variables as:

$$
\begin{aligned}
& u=\frac{u^{*}}{U_{0}}, \mathrm{v}=\frac{v^{*}}{V_{0}}, v_{w}=\frac{v_{w}{ }^{*}}{V_{0}} \mathrm{y}=\frac{V_{0} y^{*}}{v}, u_{\infty}=\frac{u_{\infty}^{*}}{U_{0}}, u_{p}=\frac{u_{p}^{*}}{U_{0}}, \\
& \theta=\left(T^{*}-T_{\infty}^{*}\right) \frac{k v}{q_{w} V_{0}}, \mathrm{C}_{R}=\frac{K_{l} v}{V_{0}^{2}}, \phi=\frac{C^{*}-C_{\infty}^{*}}{C_{w}^{*}-C_{\infty}^{*}}, \mathrm{t}=\frac{t^{*} V_{0}^{2}}{v}, G r=\frac{g \beta_{T} q_{w}}{k U_{0} V_{0}}, \\
& G m=\frac{g \beta_{C} v\left(C_{w}^{*}-C_{\infty}^{*}\right)}{U_{0} V_{0}^{2}}, M^{*}=\frac{\sigma B_{0}^{2} v}{\rho V_{0}^{2}}, \mathrm{~K}=\frac{V_{0}^{2} K^{*}}{v^{2}}, \omega=\frac{v \omega^{*}}{V_{0}^{2}} \\
& \operatorname{Pr}=\frac{K}{\rho v C_{p}}, \mathrm{R}^{*}=\frac{3 \rho C_{p} k_{1}}{4}, \mathrm{R}=\frac{4 \sigma_{1}}{k R^{*}}, \mathrm{Ec}=\frac{\mu U_{0}^{2} k}{\rho q_{w} V_{0} C_{p}}, \\
& Q s=\frac{Q s^{*} v}{\rho C_{p} V_{0}^{2}}, S \mathrm{r}=\frac{D_{T} q_{w} V_{0}}{K v^{2}\left(C_{w}^{*}-C_{\infty}^{*}\right)}
\end{aligned}
$$

On using the dimensionless quantities, equations (1) - (4) can thus be transformed as:

$$
\frac{\partial v}{\partial y}=0
$$

$$
\frac{\partial u}{\partial t}+v \frac{\partial u}{\partial y}=\frac{\partial u_{\infty}}{\partial t}+\frac{\partial^{2} u}{\partial y^{2}}+G r \theta \cos \psi+G m \phi \cos \psi+\left(M^{2}+\frac{1}{K}\right)\left(u_{\infty}-u\right)
$$

Using (7) and (8) in (3) gives,

$$
\begin{aligned}
& \frac{\partial \theta}{\partial t}+v \frac{\partial \theta}{\partial y}=\frac{1}{\operatorname{Pr}}\left(1+\frac{4 R}{3}\right) \frac{\partial^{2} \theta}{\partial y^{2}}+E c\left(\frac{\partial u}{\partial y}\right)^{2}+Q s \theta \\
& \frac{\partial \phi}{\partial t}+v \frac{\partial \phi}{\partial y}=\frac{1}{S c} \frac{\partial^{2} \phi}{\partial y^{2}}-C_{R} \phi+S r \frac{\partial^{2} \theta}{\partial y^{2}}
\end{aligned}
$$

With non - dimensional boundary conditions as:

$$
\left.\begin{array}{l}
\begin{array}{l}
u=0, v=0, \theta=0, \phi=0, \forall y \text { and } \mathrm{t}=0 \\
u=u_{p}, v=v_{w}(t), \theta^{\prime}=-1, \phi=1+\varepsilon \exp (i \omega t) \text { at } y=0 \text { when } t>0
\end{array} \\
\text { and } u=u_{\infty}=1+\varepsilon \exp (i \omega t), \theta \rightarrow 0, \phi \rightarrow 0 \text { for } \mathrm{y} \rightarrow \infty \text { when } \mathrm{t}>0
\end{array}\right\}
$$

The equation (7) suggests an asymptotic time dependent solution of $v$ : $v=v_{w}=-(1+\varepsilon \lambda \exp (i \omega t))$

\section{METHODOLOGY}

To integrate the set of non- liner, coupled system of equations (9) to (12) subject to conditions (13) and (14), we propose a multi - parameter perturbation method. Following this we take $1^{\text {st }}$ set asymptotic solutions form as:

$$
f(y, t)=f_{0}(y)+\varepsilon f_{1}(y) \exp (i \omega t)+0\left(\varepsilon^{2}\right)
$$


Where $f$ stands for $u, \Theta$ and $\varphi$.

On collecting the co-efficient of various powers of fluctuating parameter, we obtain a set of non - perturbed and perturbed system of equations as:

$\frac{d^{2} u_{0}}{d y^{2}}+\frac{d u_{0}}{d y}-M_{1} u_{0}=-M_{1}-G r \theta_{0} \cos \psi-G m \phi_{0} \cos \psi$

$\left(1+\frac{4 R}{3}\right) \frac{d^{2} \theta_{0}}{d y^{2}}+\operatorname{Pr} \frac{d \theta_{0}}{d y}+Q s \operatorname{Pr} \theta_{0}=-E c \operatorname{Pr}\left(\frac{\partial u_{0}}{\partial y}\right)^{2}$

$\frac{d^{2} \phi_{0}}{d y^{2}}+S c \frac{d \phi_{0}}{d y}-C_{R} S c \phi_{0}=-S r S c \frac{d^{2} \theta_{0}}{d y^{2}}$

$\frac{d^{2} u_{1}}{d y^{2}}+\lambda \frac{d u_{1}}{d y}-\left(M_{1}+i \omega\right) u_{1}=-\left(M_{1}+i \omega\right)-G r \theta_{1} \cos \psi-G m \phi_{1} \cos \psi$

$\left(1+\frac{4 R}{3}\right) \frac{d^{2} \theta_{1}}{d y^{2}}+\operatorname{Pr} \lambda \frac{d \theta_{1}}{d y}+(Q s-i \omega) \operatorname{Pr} \theta_{1}=-2 E c \operatorname{Pr}\left(\frac{d u_{1}}{d y}\right)\left(\frac{d u_{0}}{d y}\right)$

$\frac{d^{2} \phi_{1}}{d y^{2}}+\lambda S c \frac{d \phi_{1}}{d y}-\left(C_{R}+i \omega\right) S c \phi_{1}=-S r S c \frac{d^{2} \theta_{1}}{d y^{2}}$

With the boundary conditions follow as,

$\left.\begin{array}{l}u_{0}(0, t)=u_{p}, \theta_{0}^{\prime}(0, t)=-1, \phi_{0}(0, t)=1 \text {, when } t>0 \\ u_{0}(\infty, t)=1, \theta_{0}{ }^{\prime}(\infty, t)=0, \phi_{0}(\infty, t)=0 \text { when } t>0 \\ u_{1}(0, t)=0, \theta_{1}{ }^{\prime}(0, t)=0, \phi_{1}(0, t)=1 \text { when } t>0 \\ u_{1}(\infty, t)=1, \theta_{1}{ }^{\prime}(\infty, t)=0, \phi_{1}(\infty, t)=0 \text { when } t>0\end{array}\right\}$

We again propose a set of 2 nd part of trial solutions as:

$$
\begin{aligned}
& f_{0}(y)=f_{00}(y)+E c f_{01}(y)+o\left(E c^{2}\right) \text { and } \\
& f_{1}(y)=f_{10}(y)+E c f_{11}(y) \exp (i \omega t)+o\left(E c^{2}\right)
\end{aligned}
$$

Where $f$ stands for $\mathrm{u}, \Theta$ and $\varphi$ respectively

We also have, $E c<<1$ for an incompressible fluid.

By virtue of the $2^{\text {nd }}$ form of solutions, the non - perturbed set of equations thus governed as:

$\left(1+\frac{4 R}{3}\right) \frac{d^{2} \theta_{00}}{d y^{2}}+\operatorname{Pr} \frac{d \theta_{00}}{d y}+Q s \operatorname{Pr} \theta_{00}=0$

$\frac{d^{2} \phi_{00}}{d y^{2}}+S c \frac{d \phi_{00}}{d y}-C_{R} S c \phi_{00}=-S r S c \frac{d^{2} \theta_{00}}{d y^{2}}$

$\frac{d^{2} u_{00}}{d y^{2}}+\frac{d u_{00}}{d y}-M_{1} u_{00}=-M_{1}-G r \theta_{00} \cos \psi-G m \phi_{00} \cos \psi$

$\left(1+\frac{4 R}{3}\right) \frac{d^{2} \theta_{01}}{d y^{2}}+\operatorname{Pr} \frac{d \theta_{01}}{d y}+Q s \operatorname{Pr} \theta_{01}=\operatorname{Pr}\left(\frac{d u_{00}}{d y}\right)^{2}$

$\frac{d^{2} \phi_{01}}{d y^{2}}+S c \frac{d \phi_{01}}{d y}-C_{R} S c \phi_{01}=-S r S c \frac{d^{2} \theta_{01}}{d y^{2}}$ $\frac{d^{2} u_{01}}{d y^{2}}+\frac{d u_{01}}{d y}-M_{1} u_{01}=-G r \theta_{01} \cos \psi-G m \phi_{01} \cos \psi$

With the boundary conditions as:

$u_{00}(0, t)=u_{p}, \theta_{00}^{1}(0, t)=-1, \phi_{00}(0, t)=1$, when $t>0$

$u_{00}(\infty, t) \rightarrow 1, \theta_{00}(\infty, t) \rightarrow 0, \phi_{00}(\infty, t) \rightarrow 0$ when $\left.t>0\right\}$

$u_{01}(0, t)=0, \theta_{01}^{1}(0, t)=0, \phi_{01}(0, t)=0$, when $t>0$

$u_{01}(\infty, t) \rightarrow 0, \theta_{01}(\infty, t) \rightarrow 0, \phi_{01}(\infty, t) \rightarrow 0$ when $\left.t>0\right\}$

Again a set of perturbed equations also formed as:

$\left(1+\frac{4 R}{3}\right) \frac{d^{2} \theta_{10}}{d y^{2}}+\operatorname{Pr} \lambda \frac{d \theta_{10}}{d y}+(Q s-i \omega) \operatorname{Pr} \theta_{10}=0$
$\frac{d^{2} \phi_{10}}{d y^{2}}+\lambda S c \frac{d \phi_{10}}{d y}-\left(C_{R}+i \omega\right) S c \phi_{10}=-S r S c \frac{d^{2} \theta_{10}}{d y^{2}}$
$\frac{d^{2} u_{10}}{d y^{2}}+\lambda \frac{d u_{10}}{d y}-\left(M_{1}+i \omega\right) u_{10}=-\left(M_{1}+i \omega\right)-G r \theta_{10} \cos \psi-G m \phi_{10} \cos \psi$

$\left(1+\frac{4 R}{3}\right) \frac{d^{2} \theta_{11}}{d y^{2}}+\operatorname{Pr} \lambda \frac{d \theta_{11}}{d y}+(Q s-i \omega) \operatorname{Pr} \theta_{11}=-2 \operatorname{Pr}\left(\frac{d u_{00}}{d y}\right)\left(\frac{d u_{10}}{d y}\right)$

$\frac{d^{2} \phi_{11}}{d y^{2}}+S c \lambda \frac{d \phi_{11}}{d y}-\left(C_{R}+i \omega\right) S c \phi_{11}=-S r S c \frac{d^{2} \theta_{11}}{d y^{2}}$

$\frac{d^{2} u_{11}}{d y^{2}}+\lambda \frac{d u_{11}}{d y}-\left(M_{1}+i \omega\right) u_{11}=-G r \theta_{11} \cos \psi-G m \phi_{11} \cos \psi$

Subject to a set of boundary conditions:

$\left.\begin{array}{l}u_{10}(0, t)=0, \theta_{10}(0, t)=0, \phi_{10}(0, t)=0, \text { when } \mathrm{t}>0 \\ u_{10}(\infty, t) \rightarrow 1, \theta_{10}(\infty, t) \rightarrow 0, \phi_{10}(\infty, t) \rightarrow 0 \text { when } \mathrm{t}>0\end{array}\right\}$

$u_{11}(0, t)=0, \theta_{11}^{1}(0, t)=0, \phi_{11}(0, t)=0$, when $\mathrm{t}>0$

$u_{11}(\infty, t) \rightarrow 0, \theta_{11}(\infty, t) \rightarrow 0, \phi_{11}(\infty, t) \rightarrow 0$ when $\left.\mathrm{t}>0\right\}$

On using straight forward integration schemes, the closed form solutions for the equations (22) to (27) in real domain are finally calculated as:

$\theta_{R}(y)=\frac{1}{x_{1}} \exp \left(x_{1} y\right)+E c\left[\begin{array}{l}d_{3} \exp \left(-x_{4} y\right)+A_{7} \exp \left(-2 x_{3} y\right)+A_{8} \exp \left(-2 x_{2} y\right) \\ +A_{9} \exp \left(-2 x_{1} y\right)+A_{10} \exp \left(-\left(x_{3}+x_{2}\right) y\right) \\ +A_{11} \exp \left(-\left(x_{1}+x_{2}\right) y\right)+A_{12} \exp \left(-\left(x_{3}+x_{1}\right) y\right)\end{array}\right]$
$+2 \varepsilon E c \operatorname{Pr}\left[\begin{array}{l}\exp \left(b_{5} y\right)\left(A_{68} \cos \left(b_{6} y\right)+A_{69} \sin \left(b_{6} y\right)\right) \\ -\exp \left(-\left(x_{3}+b_{3}\right) y\right)\left(d_{31} \cos \left(b_{4} y\right)+d_{31} \sin \left(b_{4} y\right)\right) \\ -\exp \left(-\left(x_{2}+b_{3}\right) y\right)\left(d_{33} \cos \left(b_{4} y\right)+d_{34} \sin \left(b_{4} y\right)\right) \\ -\exp \left(-\left(x_{1}+b_{3}\right) y\right)\left(d_{35} \cos \left(b_{4} y\right)+d_{36} \sin \left(b_{4} y\right)\right) \\ +\exp \left(-\left(x_{3}+b_{1}\right) y\right)\left(d_{49} \cos \left(b_{2} y\right)+d_{50} \sin \left(b_{2} y\right)\right) \\ +\exp \left(-\left(x_{2}+b_{1}\right) y\right)\left(d_{51} \cos \left(b_{2} y\right)+d_{52} \sin \left(b_{2} y\right)\right) \\ +\exp \left(-\left(x_{1}+b_{1}\right) y\right)\left(d_{53} \cos \left(b_{2} y\right)+d_{54} \sin \left(b_{2} y\right)\right)\end{array}\right]$ 


$$
\begin{gathered}
\phi_{R}(y)=\left(1+d_{1}\right) \exp \left(-x_{2} y\right)-d_{1} \exp \left(-x_{1} y\right)+E c\left[\begin{array}{l}
d_{4} \exp \left(-x_{5} y\right)+A_{13} \exp \left(-x_{4} y\right)+A_{14} \exp \left(-2 x_{3} y\right) \\
+A_{15} \exp \left(-2 x_{2} y\right)+A_{16} \exp \left(-2 x_{1} y\right)+A_{17} \exp \left(-\left(x_{3}+x_{2}\right) y\right) \\
+A_{18} \exp \left(-\left(x_{2}+x_{1}\right) y\right)+A_{19} \exp \left(-\left(x_{1}+x_{3}\right) y\right)
\end{array}\right) \\
+\varepsilon \exp \left(-b_{1} y\right) \cos \left(b_{2} y\right)+\varepsilon E c S c S r\left[\begin{array}{l}
\exp \left(-b_{7} y\right)\left(d_{95} \cos \left(b_{8} y\right)+d_{96} \sin \left(b_{8} y\right)\right) \\
-\exp \left(-b_{5} y\right)\left(A_{70} \cos \left(b_{6} y\right)+A_{71} \sin \left(b_{6} y\right)\right)
\end{array}\right] \\
-2 \operatorname{Pr} \varepsilon E c S c S r\left[\begin{array}{l}
\exp \left(-\left(x_{3}+b_{3}\right) y\right)\left(A_{72} \cos \left(b_{4} y\right)+A_{73} \sin \left(b_{4} y\right)\right) \\
+\exp \left(-\left(x_{2}+b_{3}\right) y\right)\left(A_{74} \cos \left(b_{4} y\right)+A_{75} \sin \left(b_{4} y\right)\right) \\
+\exp \left(-\left(x_{1}+b_{3}\right) y\right)\left(A_{76} \cos \left(b_{4} y\right)+A_{77} \sin \left(b_{4} y\right)\right) \\
-\exp \left(-\left(x_{3}+b_{1}\right) y\right)\left(A_{78} \cos \left(b_{2} y\right)+A_{79} \sin \left(b_{2} y\right)\right) \\
-\exp \left(-\left(x_{2}+b_{1}\right) y\right)\left(A_{80} \cos \left(b_{2} y\right)+A_{81} \sin \left(b_{2} y\right)\right) \\
-\exp \left(-\left(x_{1}+b_{1}\right) y\right)\left(A_{82} \cos \left(b_{2} y\right)+A_{83} \sin \left(b_{2} y\right)\right)
\end{array}\right]
\end{gathered}
$$

$u_{R}(y)=1+A_{5} \exp \left(-x_{3} y\right)+A_{4} \exp \left(-x_{2} y\right)+A_{6} \exp \left(-x_{1} y\right)$

$$
\begin{aligned}
& +E c\left[\begin{array}{l}
d_{5} \exp \left(-x_{6} y\right)+A_{27} \exp \left(-x_{5} y\right)+A_{36} \exp \left(-2 x_{3} y\right)+A_{37} \exp \left(-2 x_{2} y\right)+A_{38} \exp \left(-2 x_{1} y\right) \\
+\left(A_{35}+A_{39}\right) \exp \left(-\left(x_{3}+x_{2}\right) y\right)+A_{40} \exp \left(-\left(x_{1}+x_{2}\right) y\right)+A_{41} \exp \left(-\left(x_{1}+x_{3}\right) y\right)
\end{array}\right] \cos \psi \\
& +\varepsilon\left[\begin{array}{l}
\exp \left(-b_{3} y\right)\left\{\operatorname{Gm} \cos \psi\left(A_{42} \cos \left(b_{4} y\right)-A_{43} \sin \left(b_{4} y\right)\right)-\cos \left(b_{4} y\right)\right\} \\
-\exp \left(-b_{1} y\right) G m \cos \psi\left(A_{42} \cos \left(b_{2} y\right)-A_{43} \sin \left(b_{2} y\right)\right)+1
\end{array}\right]
\end{aligned}
$$$$
+\varepsilon E c \cos \psi\left[\begin{array}{l}
\exp \left(-b_{9} y\right)\left(A_{100} \cos \left(b_{10} y\right)+A_{101} \sin \left(b_{10} y\right)\right) \\
-\exp \left(-b_{7} y\right)\left(A_{84} \cos \left(b_{8} y\right)+A_{85} \sin \left(b_{8} y\right)\right) \\
-\exp \left(-b_{5} y\right)\left(A_{86} \cos \left(b_{6} y\right)+A_{87} \sin \left(b_{6} y\right)\right) \\
-\exp \left(-\left(x_{3}+b_{3}\right) y\right)\left(A_{88} \cos \left(b_{4} y\right)+A_{89} \sin \left(b_{4} y\right)\right) \\
-\exp \left(-\left(x_{2}+b_{3}\right) y\right)\left(A_{90} \cos \left(b_{4} y\right)+A_{91} \sin \left(b_{4} y\right)\right) \\
-\exp \left(-\left(x_{1}+b_{3}\right) y\right)\left(A_{92} \cos \left(b_{4} y\right)+A_{93} \sin \left(b_{4} y\right)\right) \\
-\exp \left(-\left(x_{3}+b_{1}\right) y\right)\left(A_{94} \cos \left(b_{2} y\right)+A_{95} \sin \left(b_{2} y\right)\right) \\
-\exp \left(-\left(x_{2}+b_{1}\right) y\right)\left(A_{96} \cos \left(b_{2} y\right)+A_{97} \sin \left(b_{2} y\right)\right) \\
-\exp \left(-\left(x_{1}+b_{1}\right) y\right)\left(A_{98} \cos \left(b_{2} y\right)+A_{99} \sin \left(b_{2} y\right)\right)
\end{array}\right]
$$

\section{SOME SIGNIFICANT QUANTITIES OF ENGINEERING INTEREST}

\subsection{Skin friction at the plate:}

The real part of the non-dimensional skin-friction coefficient at the plate is obtained

as:

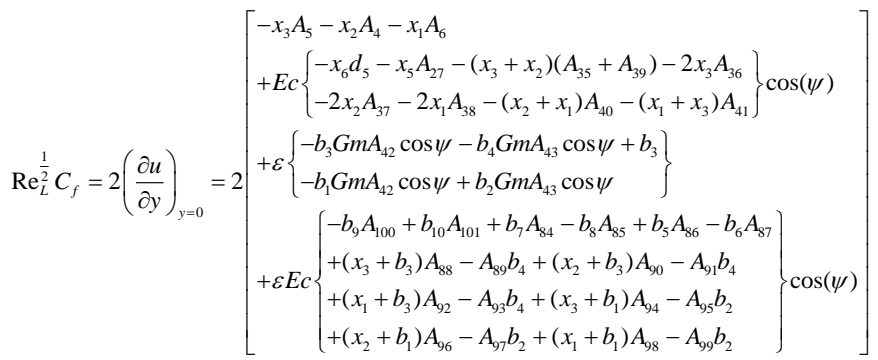

where $\mathrm{C}_{f}=\frac{2\left(\tau_{\omega}\right)_{y=0}}{\rho v_{0}^{2}}$

5.2. Rate of heat transfer coefficient: The real part of the rate of heat transfer coefficient in terms of the Nusselt number is given as:

$$
\operatorname{Re}_{L}^{-1} N_{u}=-\left(\frac{\partial \theta}{\partial y}\right)_{y=0}=\left[\begin{array}{l}
1+E c\left\{\begin{array}{l}
x_{4} d_{3}+x_{3} A_{7}+2 x_{2} A_{8}+2 x_{1} A_{9}+\left(x_{3}+x_{2}\right) A_{10} \\
+\left(x_{2}+x_{1}\right) A_{11}+\left(x_{1}+x_{3}\right) A_{12}
\end{array}\right\} \\
-2 \operatorname{Pr} E c \varepsilon\left\{\begin{array}{l}
-b_{5} A_{68}+b_{6} A_{69}++\left(x_{3}+b_{3}\right) d_{31}-d_{32} b_{4} \\
+\left(x_{2}+b_{3}\right) d_{33}-d_{34} b_{4}+\left(x_{1}+b_{3}\right) d_{35}-d_{36} b_{4} \\
-\left(x_{3}+b_{1}\right) d_{49}+d_{50} b_{2}-\left(x_{2}+b_{1}\right) d_{51}+d_{52} b_{2} \\
-\left(x_{1}+b_{1}\right) d_{54} b_{2}
\end{array}\right\}
\end{array}\right]
$$

where $\mathrm{Nu}=\frac{-k\left(\frac{\partial T}{\partial y}\right)_{y=0}}{T_{w}-T_{\infty}}$

\subsection{Rate of mass transfer coefficient:}

The mass transfer coefficient in terms of the Sherwood number as:

where Sh $=\frac{-D_{M}\left(\frac{\partial C}{\partial y}\right)_{y=0}}{C_{w}-C_{\infty}}$

$\operatorname{Re}_{x}^{-1} S_{h}=-\left(\frac{\partial \phi}{\partial y}\right)_{y=0}=\left[\begin{array}{l}x_{2}\left(1+d_{1}\right)+x_{1} d_{1}-E c\left\{\begin{array}{l}-x_{5} d_{4}-x_{4} A_{13}-2 x_{3} A_{14}-2 x_{2} A_{15}-2 x_{1} A_{16} \\ -\left(x_{3}+x_{2}\right) A_{17}-\left(x_{2}+x_{1}\right) A_{18}-\left(x_{1}+x_{3}\right) A_{19}\end{array}\right\} \\ +\varepsilon a_{5}-\operatorname{ScSrEc} \varepsilon\left\{-a_{16} d_{95}+a_{17} d_{96}+a_{14} A_{70}-a_{15} A_{71}\right\} \\ +2 \operatorname{Pr} \operatorname{ScS} \operatorname{SrEc}\left\{\begin{array}{l}-\left(x_{3}+a_{10}\right) A_{72}-a_{11} A_{73}-\left(x_{2}+a_{10}\right) A_{74}+A_{74} a_{11} \\ -\left(x_{1}+a_{10}\right) A_{76}+A_{77} a_{11}+\left(x_{3}+a_{5}\right) A_{78}-A_{79} a_{7} \\ +\left(x_{2}+a_{5}\right) A_{80}-A_{81} a_{7}+\left(x_{1}+a_{5}\right) A_{82}-A_{83} a_{7}\end{array}\right\}\end{array}\right]$

\section{RESULTS AND DISCUSSION}

To investigate the influence of various physical parameters on the flow variables, numerical simulations have been made on the flow properties by assigning appropriate values of the governed physical parameters such as heat source parameter $(Q s)$, Eckert number $(E c)$, thermal radiation parameter $(R)$, chemical reaction parameter $\left(C_{R}\right)$, Schmidt number $(S c)$, Soret number $(S r)$, magnetic field parameter $(M)$, permeability parameter $(K)$, plate inclination parameter $(\psi)$, thermal Grashof number $(G r)$ and solutal Grashof number $(G m)$. The Prandtl number is taken as 7.0 which represent water at temperature $25^{\circ} \mathrm{C}$ or $298 \mathrm{~K}$ under $1 \mathrm{~atm}$ pressure. Again throughout this study, water is considered to be a primary fluid (solvent) and the values of Schmidt (Sc) number of secondary species (solute) such as Hydrogen is taken as 0.22. Figure 2, 3, 4, and 5 illustrate the effect of Eckert number, magnetic field parameter, heat source parameter and thermal radiation parameter on non - dimensional temperature profile $\left(\theta_{R}, y\right)$ for a set of fixed values of $\lambda=0.5, u_{p}=1.0, \operatorname{Pr}=7.0, G r=0.5, E=0.001, S r=0.5$, $S c=0.22, \quad C_{R}=0.5, \omega=7.857143, t=0.5, \quad \psi=0.7857$, (Fig.2, 3, 4, 5) $E c=0.01$ (Fig.3， 4, 5), $M=1.001$ (Fig.2， 4, 5), $Q s=0.1$ (Fig.2), $Q s=0.5$ (Fig.3),$\quad Q s=0.7$ (Fig.5),$\quad R=1.5$ (Fig.2), $\quad R=0.5 \quad$ (Fig.3), $R=0.3$ (Fig.4) $\quad K=1.2$ (Fig.2, 4 ), $\quad K=0.5$ (Fig.3),$\quad K=0.2$ (Fig.5), $G m=0.3$ (Fig.2), $G m=0.6$ (Fig.3, 4), $G m=0.1$ (Fig.4). In figure 2, a parametric increase in the values of Eckert number $(E c)$ is found to increase the temperature of the fluid particles. As temperature fluxes generate due to the heat produced in the medium by the dissipation of energy created by internal friction, this is seen to increase the temperature within the thermal boundary layer. On the other hand, as the magnetic field parameter generates a resistive force in terms of Lorentz force, which decreases the energy of the fluid particles, the loss in energy is thus converted in to heat energy, which rises temperature near the plate surface. Againan increase in values of $Q s$ is found to increase the temperature of fluid particles near the plate surface. This is due to the fact that, the temperature fluxes inside the thermal boundary layer get rises, results of which, the values of $\theta_{R}$ increases but decreases gradually with increase in values of normal distances. As the temperature gradient inside the thermal boundary layer rises due to presence of thermal radiation parameter, an increase in values of fluid temperature is thus observed. 


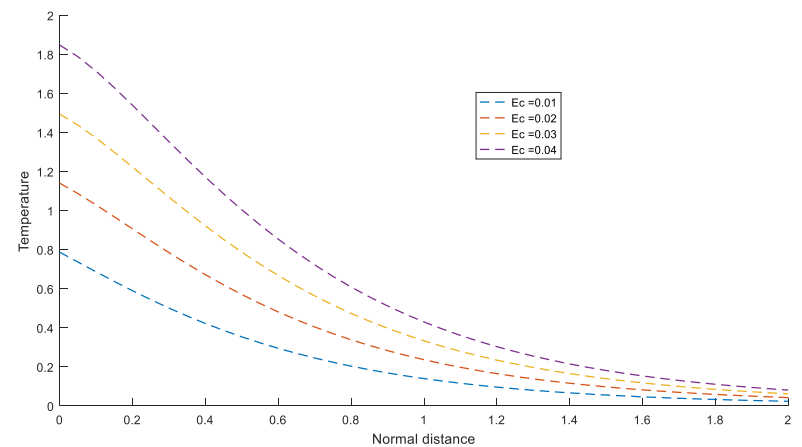

Fig. 2 Temperature $\left(\theta_{R}\right)$ versus Normal distance (y) for arbitrary change in values of Eckert number $(E c)$.

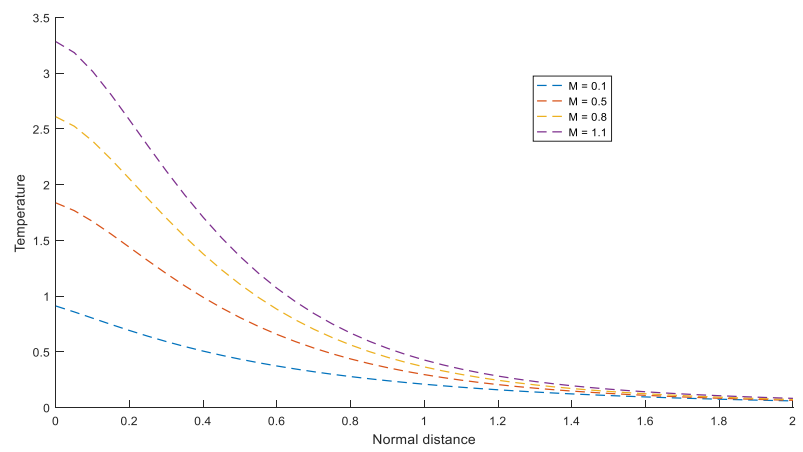

Fig. 3 Temperature ( $\left.\theta_{R}\right)$ versus Normal distance (y) for arbitrary change in values of Magnetic field parameter $(M)$.

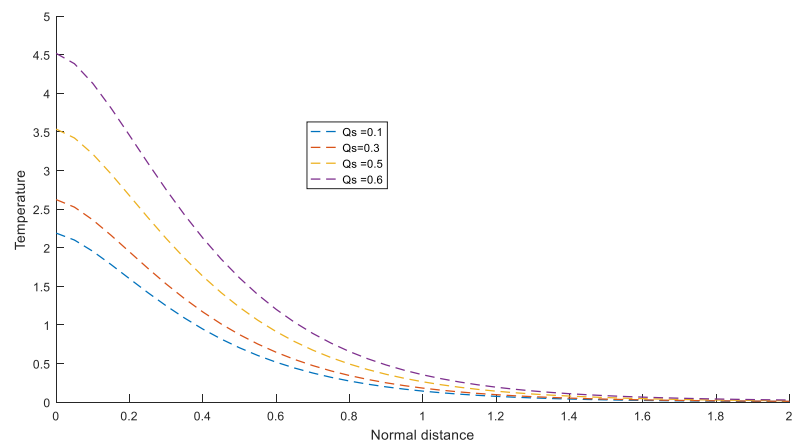

Fig. 4 Temperature $\left(\theta_{R}\right)$ versus Normal distance $(y)$ for arbitrary change in values of Heat source parameter $(Q s)$.

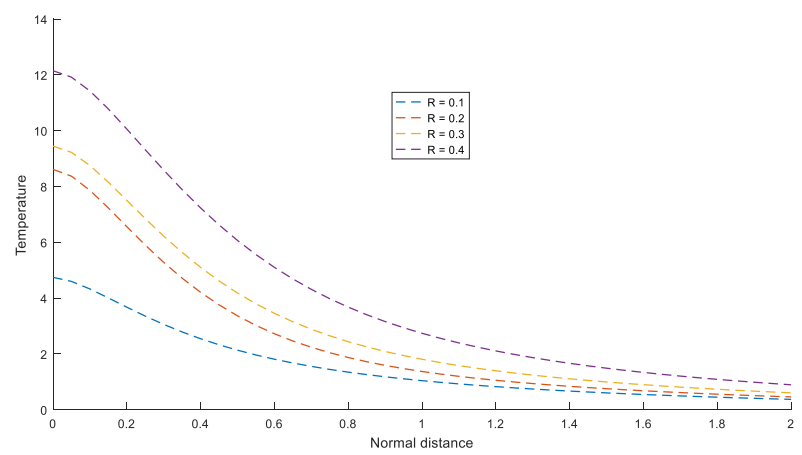

Fig. 5 Temperature $\left(\theta_{R}\right)$ versus Normal distance (y) for arbitrary change in values of Radiation parameter $(R)$.

In figure $(6,7,8,9,10,11)$ the effect of heat source parameter $(Q s)$, Eckert number $(E c)$, magnetic field parameter $(M)$ chemical reaction parameter $\left(C_{R}\right)$, Schmidt number $(S c)$ and Soret number $(S r)$ on the concentration profiles has been depicted for a set of fixed values of $u_{p}=1.0, \operatorname{Pr}=7.0, \varepsilon=0.001, \infty=7.857143, t=0.5, \psi=0.7857$ (Fig. 6, 7, 8, 9, 10, 11), $\lambda=0.5$ (Fig 6, 7, 8, 10, 11), $\lambda=0.2$ (Fig.9) , $K=0.3$, (fig.6) $K=0.1$ (Fig.7, 11 ), $K=0.5 \quad$ (Fig.9), $K=0.7$ (Fig.8), $K=0.4 \quad$ (Fig.10), $G m=0.2$, (Fig.6, 9, 10), $G m=0.5$ (Fig.7, 11), $G m=1.0$ (Fig.8) $G r=0.3$, (Fig.6) $G r=2.0$ (Fig.8, 10), $G r=0.5$ (Fig.7, 11), Gr=0.1(Fig.9), $S c=0.22$, (Fig. 6, 7, 8, 9, 11), $C_{R}=0.5$, (Fig. 6, 7, 8, 10, 11), $S r=0.5$, (Fig.6, 10), $S r=0.7$ (Fig.7), $S r=2.1$ (Fig.8), $S r=0.1$ (Fig.9), Ec=0.01(Fig.6, 8, 9, 10, 11), $Q s=0.1$ (Fig.7, 11), $Q s=0.4$ (Fig.9, 10), $Q s=1.1$ (Fig.8). Due to positive increase in values of $Q s, E c, S r$ and $M$, the mass fluxes inside the solutal boundary layer increases. This help in increasing the molar concentrations near the plate region and this increases the concentrations of the fluid particles near the plate. On the other hand as the process of diffusion of mass increases due to presence of $C_{R}$ and Sc, the thickness of the solutal boundary layer diminishes and molar concentration of the constituents gets thinner thereby decreasing the species concentration near plate surface.

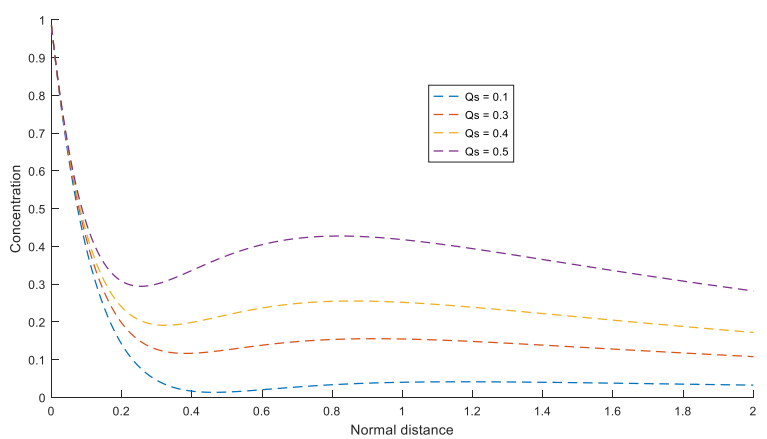

Fig. 6 Concentration $\left(\phi_{R}\right)$ versus Normal distance $(y)$ for arbitrary change in values of Heat source parameter $(Q s)$.

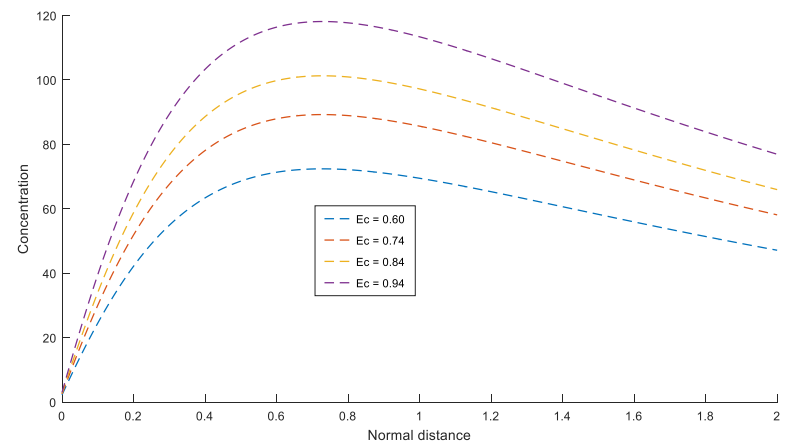

Fig. 7 Concentration $\left(\phi_{R}\right)$ versus Normal distance $(y)$ for arbitrary change in values of Eckert number $(E c)$.

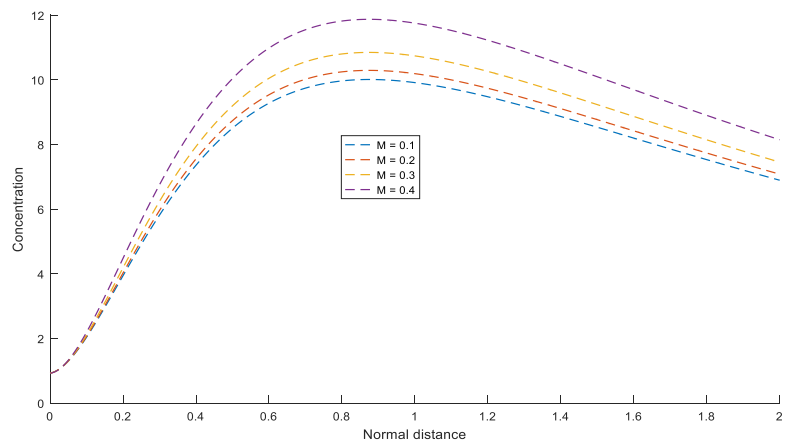

Fig. 8 Concentration $\left(\phi_{R}\right)$ versus Normal distance $(y)$ for arbitrary change in values of Magnetic field parameter $(M)$. 


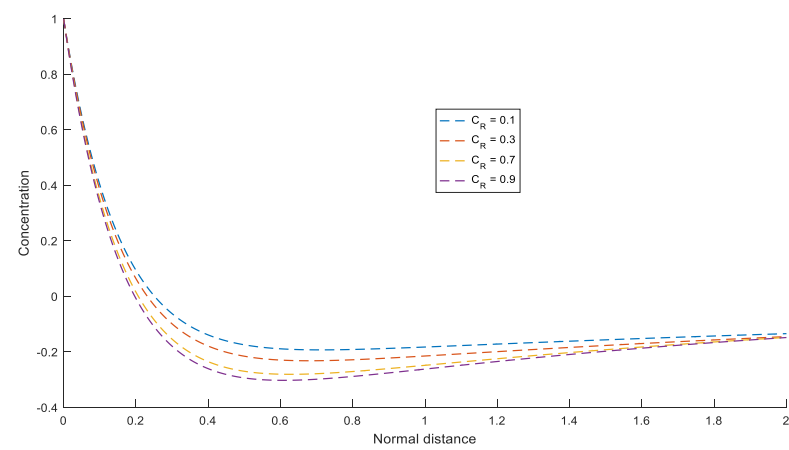

Fig. 9 Concentration $\left(\phi_{R}\right)$ versus Normal distance $(y)$ for arbitrary change in values of Chemical reaction $\left(C_{R}\right)$.

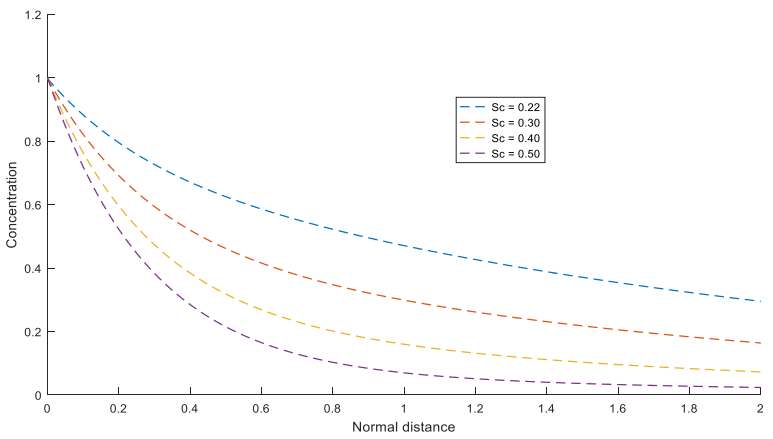

Fig.10 Concentration $\left(\phi_{R}\right)$ versus normal distance $(y)$ for arbitrary change in values of Schmidt Number $(S c)$.

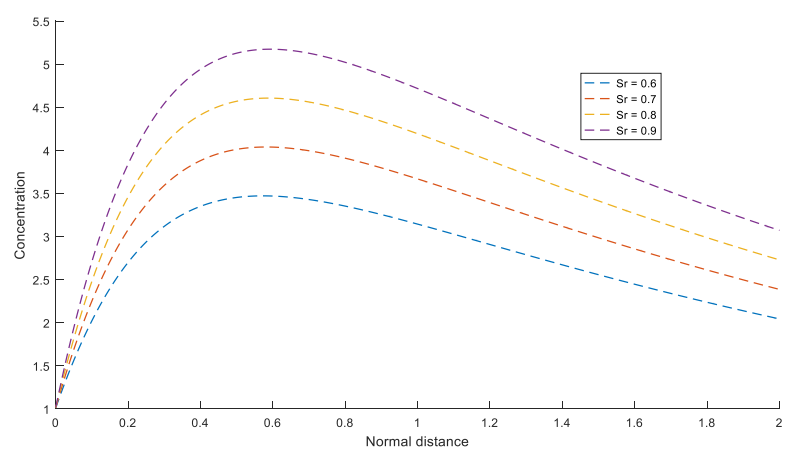

Fig. 11 Concentration $\left(\phi_{R}\right)$ versus Normal distance $(y)$ for arbitrary change in values of Soret number $(\mathrm{Sr})$.

In figure $(12,13,14,15,16,17,18,19)$, the influence of heat source parameter $(Q s)$, magnetic field parameter $(M)$, permeability parameter $(K)$, thermal radiation parameter $(R)$, Soret number $(S r)$, chemical reaction parameter $\left(C_{R}\right)$, plate inclination parameter $(\psi)$ and Eckert number $(E c)$ on the axial velocity profile $\left(u_{R}, y\right)$ is depicted for a set of fixed assigned values of $\lambda=0.5, u_{p}=1.0, \operatorname{Pr}=7.0, \varepsilon=0.001$, $E c=0.01, S c=0.22, \psi=0.7857, t=0.5, \omega=7.857143$ (Fig. 12,13, 14, 15, 16, 17, 18 and 19), $M=1.001$ (Fig.12, 14, 15, 16, 17, 18, and 19), $K=0.5$ (Fig.16), $K=0.2$ (Fig.13), $K=0.1$ (Fig.12, 15, 17), K=0.6 (Fig.18, 19), $Q s=0.6$ (Fig.13 and 14), $Q s=0.1$ (Fig.15), $Q s=1.1$ (Fig.16 and 17), $Q s=0.5$ (Fig.18 and 19), $G r=0.5$ (Fig.12, 15, 18), Gr=0.4 (Fig.13, and 14), $G r=0.9$ (Fig. 17, 19), $G r=0.6$ (Fig.16), $S r=0.5$ (Fig. 12, 15, 18, 19), $S r=0.6$ (Fig.13, 14), $S r=0.2$ (Fig.17), Gm=0.3 (Fig. 12), $G m=0.2$ (Fig. 13, 14), $G m=0.6$ (Fig.16), $G m=0.5$ ( Fig.15, 18), $G m=0.9$ (Fig.17,19), $C_{R}=0.5$ (Fig. 12, 13, 14, 15, 16, 18 and 19), $E c=0.001$ (Fig.12, 13, 14, 15, 16,17 , and 18). It is clearly observed from the figures that, the axial motion of the fluid particles along the plate boosted due to positive response of $Q s, K, R, S r$ and $E c$ while it is being disrupted due to a set of positive increment of $M, C_{R}$ and $\psi$. As the thermal buoyancy force and solutal buoyancy forces increase due to the presence of $Q s$ and $S r$ respectively, the axial flow rate of the fluid particles get accelerated thus increases the velocity of the flow. Again, due to increase in permeability parameter, the size of the solid void increases, this reduces the resistive phenomenon of the medium and help in accelerating the flow rate. The influence of the applied magnetic field on the axial motion generates a resistive force called Lorentz force which acts against the buoyancy force and creates an obstacle to the axial flow rate, this slowed down the motion there by reducing the fluid velocity. The positive increase in chemical reaction parameter is to decrease the molar concentration of fluid species due to transmission of fluid constituent from higher concentration zone (plate region) to lower concentration zone (free steam region). This thinning of concentration near the plate region discourages the mass buoyancy forces which in turn seen to reduce the axial motion. Again, a rise in plate inclination parameter expectedly minimizes the effect of both the thermal as well as mass buoyancy forces. This lowering in the effect of buoyancy forces seen to decelerate the flow rate and thus impede the values of the main velocity.

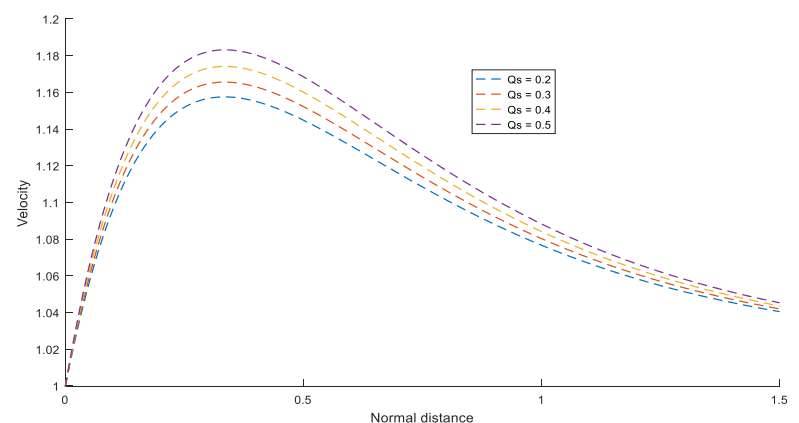

Fig. 12 Velocity $\left(u_{R}\right)$ versus Normal distance $(y)$ for arbitrary change in values of Heat source parameter $(Q s)$.

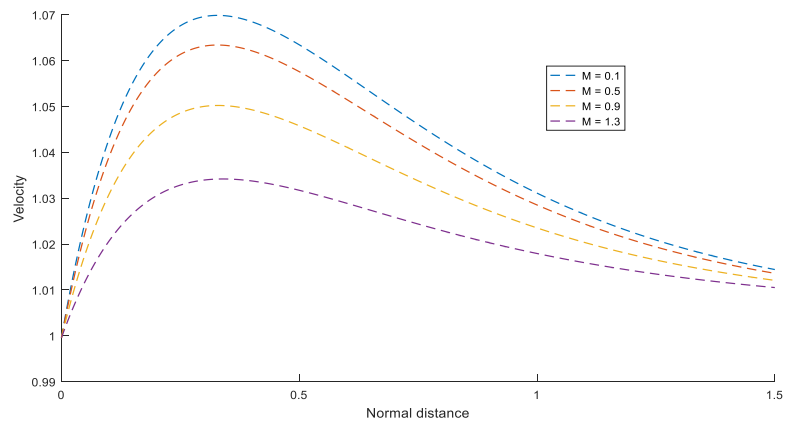

Fig. 13 Velocity $\left(u_{R}\right)$ versus Normal distance $(y)$ for arbitrary change in values of magnetic field parameter $(M)$.

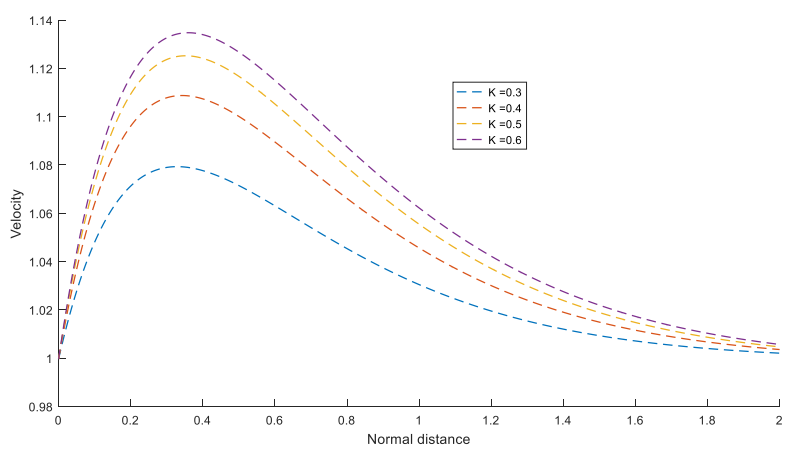

Fig. 14 Velocity $\left(u_{R}\right)$ versus Normal distance $(y)$ for arbitrary change in values of Permeability parameter $(K)$. 


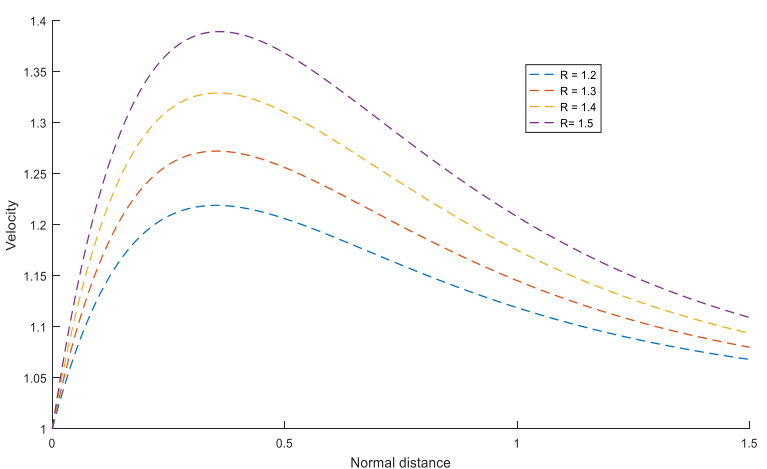

Fig. 15 Velocity $\left(u_{R}\right)$ versus Normal distance $(y)$ for arbitrary change in values of Thermal radiation $(R)$.

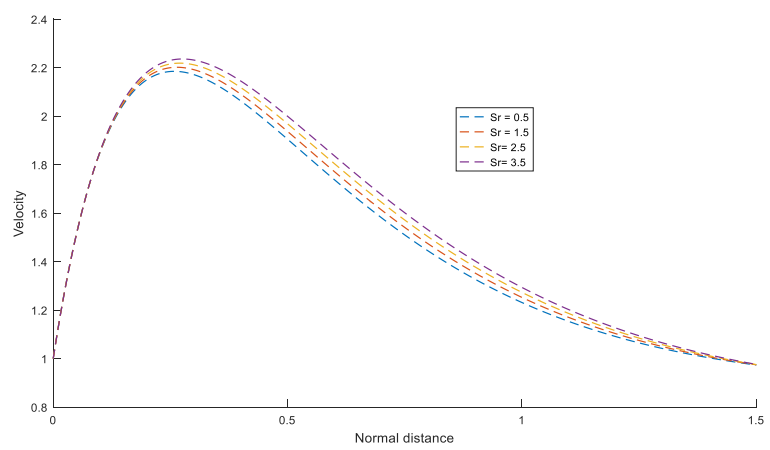

Fig. 16 Velocity $\left(u_{R}\right)$ versus Normal distance $(y)$ for arbitrary change in values of Soret number $(\mathrm{Sr})$.

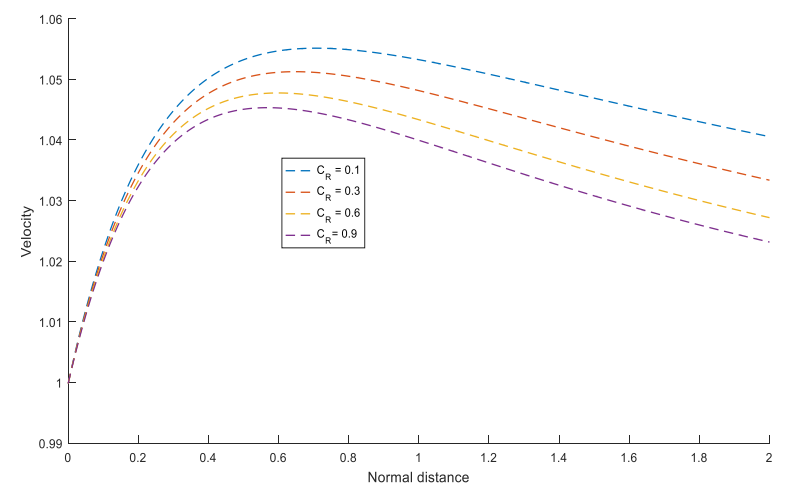

Fig. 17 Velocity $\left(u_{R}\right)$ versus Normal distance $(y)$ for arbitrary change in values of Chemical reaction $\left(C_{R}\right)$.

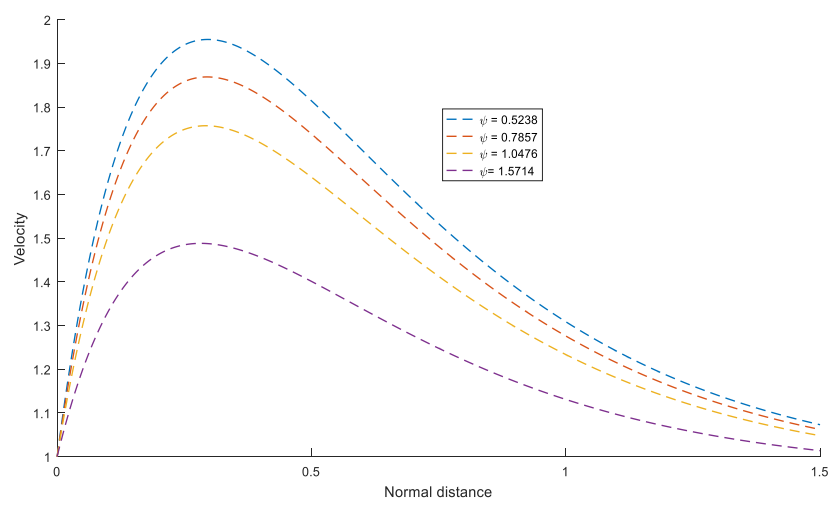

Fig. 18 Velocity $\left(u_{R}\right)$ versus Normal distance $(y)$ for arbitrary change in values of Plate inclination $(\psi)$.

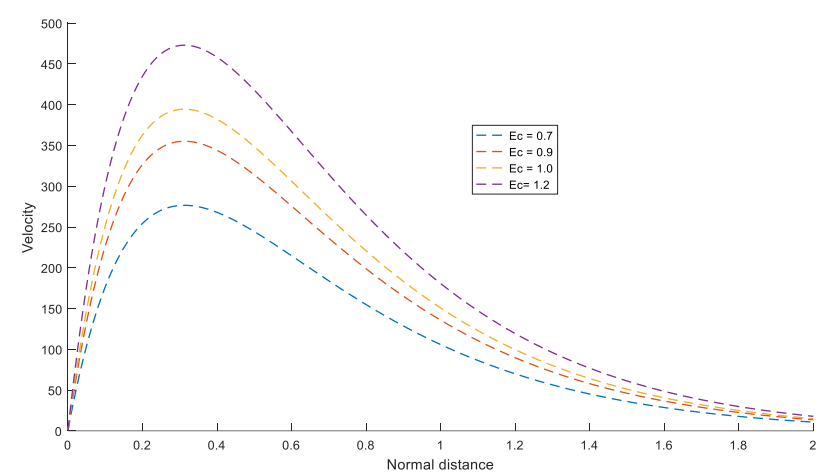

Fig. 19 Velocity $\left(u_{R}\right)$ versus Normal distance $(y)$ for arbitrary change in values of Eckert number $(E c)$.

The influence of magnetic field parameter $(M)$, Soret number $(S r)$, thermal Grashof number $(G r)$, solutal Grashof number $(G m)$, permeability parameter $(K)$, Eckert number $(E c)$ and uniform plate velocity parameter $\left(u_{p}\right)$ on skin- friction $\left(C_{f}\right)$, Nusselt number $(\mathrm{Nu})$ and Sherwood number $(S h)$ are demonstrated in table 1. It is clearly observed from this table that, the skin-friction, Nusselt and Sherwood numbers decrease due to increase in values of magnetic field parameter $(M)$, while a reverse trend is observed in case of the presence of thermal Grashof number.

Table 1 Skin-friction $\left(C_{f}\right)$, Nusselt number $(N u)$, Sherwood number $(S h)$ against time for arbitrary variation of magnetic field parameter $(M)$, Soret number $(\mathrm{Sr})$, Grashof number $(\mathrm{Gr})$, solutal Grashof number $(\mathrm{Gm})$, permeability parameter $(K)$, Eckert number $(E c)$ and uniform plate velocity $\left(u_{p}\right)$.

\begin{tabular}{|c|c|c|c|c|c|c|c|c|c|}
\hline$M$ & $\mathrm{Sr}$ & $G r$ & $G m$ & $K$ & $E c$ & $\mathrm{u}_{\mathrm{p}}$ & $R_{L}^{\frac{1}{2}} C_{f}$ & $\operatorname{Re}_{L}^{-1} N_{u}$ & $\operatorname{Re}_{L}^{-1} S_{h}$ \\
\hline 0.3 & 0.5 & 0.5 & 0.5 & 0.1 & 0.01 & 1.0 & 9.2577 & -144.58 & 4.1076 \\
\hline 0.4 & 0.5 & 0.5 & 0.5 & 0.1 & 0.01 & 1.0 & 9.2151 & -142.63 & 4.0042 \\
\hline 0.5 & 0.5 & 0.5 & 0.5 & 0.1 & 0.01 & 1.0 & 9.1489 & -140.22 & 3.8814 \\
\hline 0.6 & 0.5 & 0.5 & 0.5 & 0.1 & 0.01 & 1.0 & 9.0561 & -137.40 & 3.7451 \\
\hline 1.001 & 0.3 & 0.5 & 0.5 & 0.1 & 0.01 & 1.0 & 5.8622 & -147.176 & 0.4145 \\
\hline 1.001 & 0.4 & 0.5 & 0.5 & 0.1 & 0.01 & 1.0 & 5.7779 & -147.172 & 1.7954 \\
\hline 1.001 & 0.5 & 0.5 & 0.5 & 0.1 & 0.01 & 1.0 & 5.6936 & -147.169 & 3.1763 \\
\hline 1.001 & 0.6 & 0.5 & 0.5 & 0.1 & 0.01 & 1.0 & 5.6093 & -147.164 & 4.5571 \\
\hline 1.001 & 0.5 & 0.3 & 0.5 & 0.1 & 0.01 & 1.0 & 2.4425 & -131.23 & 3.1742 \\
\hline 1.001 & 0.5 & 0.4 & 0.5 & 0.1 & 0.01 & 1.0 & 3.0294 & -139.05 & 3.1752 \\
\hline 1.001 & 0.5 & 0.5 & 0.5 & 0.1 & 0.01 & 1.0 & 3.6147 & -147.16 & 3.1763 \\
\hline 1.001 & 0.5 & 0.6 & 0.5 & 0.1 & 0.01 & 1.0 & 4.1984 & -155.59 & 3.1773 \\
\hline 1.001 & 0.5 & 0.5 & 0.3 & 0.1 & 0.01 & 1.0 & 8.9128 & -127.81 & 2.263 \\
\hline 1.001 & 0.5 & 0.5 & 0.4 & 0.1 & 0.01 & 1.0 & 7.3573 & -137.32 & 2.714 \\
\hline 1.001 & 0.5 & 0.5 & 0.5 & 0.1 & 0.01 & 1.0 & 5.6936 & -147.16 & 3.1763 \\
\hline 1.001 & 0.5 & 0.5 & 0.6 & 0.1 & 0.01 & 1.0 & 3.9188 & -157.35 & 3.6548 \\
\hline 1.001 & 0.5 & 0.5 & 0.5 & 0.3 & 0.01 & 1.0 & 16.3984 & 88.96 & -4.364 \\
\hline 1.001 & 0.5 & 0.5 & 0.5 & 0.4 & 0.01 & 1.0 & 17.9276 & 58.64 & -3.9467 \\
\hline 1.001 & 0.5 & 0.5 & 0.5 & 0.5 & 0.01 & 1.0 & 19.0363 & 46.74 & -3.7885 \\
\hline 1.001 & 0.5 & 0.5 & 0.5 & 0.6 & 0.01 & 1.0 & 19.8748 & 40.62 & -3.709 \\
\hline 1.001 & 0.5 & 0.5 & 0.5 & 0.1 & 0.3 & 1.0 & 2.9716 & -4.444 & 200.60 \\
\hline 1.001 & 0.5 & 0.5 & 0.5 & 0.1 & 0.4 & 1.0 & 2.7499 & -5.9257 & 268.69 \\
\hline 1.001 & 0.5 & 0.5 & 0.5 & 0.1 & 0.5 & 1.0 & 2.5281 & -7.4075 & 336.77 \\
\hline 1.001 & 0.5 & 0.5 & 0.5 & 0.1 & 0.6 & 1.0 & 2.3064 & -8.8891 & 404.85 \\
\hline 1.001 & 0.5 & 0.5 & 0.5 & 0.1 & 0.01 & 0.1 & 8.0813 & 25.1399 & -8.2415 \\
\hline 1.001 & 0.5 & 0.5 & 0.5 & 0.1 & 0.01 & 0.2 & 7.6016 & 26.2337 & -8.2399 \\
\hline 1.001 & 0.5 & 0.5 & 0.5 & 0.1 & 0.01 & 0.3 & 7.1222 & 27.3525 & -8.2382 \\
\hline 1.001 & 0.5 & 0.5 & 0.5 & 0.1 & 0.01 & 0.4 & 6.6431 & 28.4962 & -8.2366 \\
\hline
\end{tabular}


Again it is found that, skin-friction and Nusselt number decrease but Sherwood number increases due to increase in Soret number $(\mathrm{Sr})$. It is also clearly observed that, the Nusselt number and Sherwood number increase, while skin-friction decreases due to increase in the values of solutal Grashof number $(\mathrm{Gm})$. The increase in permeability parameter $(K)$ is seen to increase the skin-friction but a reverse trend is observed in case of Nusselt and Sherwood numbers. Table1 also reflects that Nusselt number and Sherwood number increase while skin-friction decreases due to increase in values of Eckert number $(E c)$.

\section{CONCLUSIONS}

A theoretical study on heat and mass transfer analysis on megnetohydrodynamic (MHD) mixed convection flow of an incompressible viscous fluid in presence of thermo-diffusion along with first order chemical reaction flowing past an inclined plate is made. The significant outcome of the study is as follows:

(a) The temperature field increases due to increase in values of Eckert number, Magnetic field parameter, heat source parameter and thermal radiation parameter.

(b) The concentration field increases due to increase in values of heat source parameter, Eckert number, magnetic field parameter and Soret number, while concentrations decrease due to rise in values of chemical reaction parameter and Schmidt number.

(c) The velocity field increases due to increase in values of heat source parameter, permeability parameter, thermal radiation parameter, Soret number and Eckert number, while it decreases due to step up in values of magnetic field parameter, chemical reaction parameter, and plate inclination parameter.

(d) The coefficient of skin-friction increases due to increase in values of thermal Grashof number and permeability parameter but the surface friction decreases due to increase in magnetic field parameter, Soret number, solutal Grashof number, Eckert number and plate velocity parameter.

(e) Due to increase in magnetic field parameter, Soret number and plate velocity parameter, the Nssult number is found increasing, while an enhancement in thermal as well as solutal Grashof number, the heat transfer rate is found decreasing.

(f) The step-up in values of Soret number, Grashof number, permeability parameter, Eckert number and plate velocity parameter accelerates the mass transfer rates, while decrease with rise in magnetic field parameter.

\section{ACKNOWLEDGEMENT}

The authors extend their heartfelt thanks to the Reviewers and the Editor for providing valuable comments / suggestions to improve the quality of the paper.

\section{NOMENCLATURE}

$B_{0} \quad$ Strength of the applied magnetic field(tesla)

$\vec{B} \quad$ Total magnetic field (tesla)

$C_{p} \quad$ Specific heat at constant pressure (J/kg.K)

$C^{*} \quad$ Species concentration $\left(\mathrm{mol} / \mathrm{m}^{3}\right)$

$C_{R} \quad$ Chemical reaction parameter

$C_{w}^{*} \quad$ Wall Species concentration (Dimensional) $\left(\mathrm{mol} / \mathrm{m}^{3}\right)$

$C_{\infty}^{*} \quad$ Species concentration in the free stream $\left(\mathrm{mol} / \mathrm{m}^{3}\right)$

$\begin{array}{ll}D_{M} & \text { Mass diffusivity co-efficient }\left(\mathrm{m}^{2} / \mathrm{s}\right) \\ E_{c} & \text { Eckert number } \\ G m & \text { Grashof number for mass transfer } \\ G r & \text { Grashof number for heat transfer } \\ g & \text { Acceleration due to gravity }\left(\mathrm{m} / \mathrm{s}^{2}\right) \\ K & \text { Permeability parameter }\left(\mathrm{m}^{2}\right) \\ K_{l} & \text { Thermal diffusion ratio } \\ K_{T} & \text { Thermal diffusion coefficient }\left(\mathrm{m}^{2} / \mathrm{s}\right) \\ M & \text { Magnetic field parameter (square of the Hartmann } \\ p^{*} & \text { number) } \\ P_{r} & \left.\left.\text { Fluid pressure (Pascal (kg/(ms }{ }^{2}\right)\right) \\ Q_{s} & \text { Prandtl number } \\ R & \text { Heat generation (source) parameter }\left(\mathrm{W} / \mathrm{m}^{3} \mathrm{~K}\right) \\ \mathrm{Re}_{x}^{1 / 2} C_{f} & \text { Thermal radiation } \\ \mathrm{Re}_{x}^{-1} N_{u} & \text { Real part of non-dimensional skin friction coefficient } \\ \operatorname{Re}_{x}^{-1} S_{h} & \text { Nusselt number (Real part) } \\ S_{c} & \text { Sherwood number (Real part) } \\ S_{r} & \text { Schmidt number } \\ T_{m}^{*} & \text { Soret number } \\ T^{*} & \text { Mean fluid temperature (dimensional) }(K) \\ T_{\infty}^{*} & \text { Fluid temperature }(K) \\ u_{\infty} & \text { Temperature in the free stream } \\ u_{p} & \text { Free Stream velocity ( m/s) } \\ \left(u^{*}, v^{*}\right) & \text { Uniform plate velocity (m/s) } \\ (u, v) & \text { Velocity components (dimensional) } \\ U_{0} & \text { Melocity components (non-dimensional) } \\ V_{0} & \text { Mean plate suction velocity }(\mathrm{m} / \mathrm{s}) \\ \left(x^{*}, y^{*}\right) & \text { Cartesian Co-ordinate (dimensional) } \\ (x, y) & \text { Cartesian Co-ordinate (non-dimensional) } \\ & \end{array}$

\section{Greek Symbols}

$\begin{array}{ll}\beta_{C} & \text { Solutal volume expansion co-efficient }(1 / \mathrm{Kmol}) \\ \beta_{T} & \text { Thermal volume expansion co-efficient }(1 / \mathrm{K}) \\ \lambda & \text { Amplitude of asymptotic oscillation } \\ \sigma & \text { Stress tensor }\left(\mathrm{N} / \mathrm{m}^{2}\right) \\ \theta & \text { Non dimensional temperature } \\ \phi & \text { Non dimensional Species Concentration } \\ \psi & \text { Plate inclination (radian) } \\ v & \text { Kinematic Co-efficient of viscosity }\left(\mathrm{m}^{2} / \mathrm{s}\right) \\ \mu & \text { Dynamic viscosity }(\mathrm{kg} /(\mathrm{m} . \mathrm{s})) \\ \tau & \text { Viscous stress tensor }\left(\text { poiseuille }\left(\mathrm{N} . \mathrm{s} / \mathrm{m}^{2}\right)\right) \\ v^{*} & \text { Suction velocity }(\mathrm{m} / \mathrm{s}) \\ \rho & \text { Fluid density }\left(\mathrm{kg} / \mathrm{m}^{3}\right) \\ \rho_{\infty} & \text { Free stream fluid density }\left(\mathrm{kg} / \mathrm{m}^{3}\right) \\ \text { ubscripts } & \\ \text { Conditions on the wall } \\ \text { Free stream condition }\end{array}$

\section{REFERENCES}

Ahmed, N., Sengupta, S., and Datta, D., 2013, “An exact analysis for MHD free convection mass transfer flow past an oscillating plate embedded in a porous medium with Soret effect," Chem. Engg. Comm., 200(4), 494-513. 
Ahmed, S., Zueco, J., Luis, M., and González, L., 2017, "Effects of Chemical Reaction, Heat and Mass Transfer and Viscous Dissipation Over a MHD Flow in a Vertical Porous Wall using Perturbation Method," International Journal of Heat and Mass Transfer.,104,409418.

https ://d oi.org/10.1016/j.ijheatmasstransfer.2016.07.076

Alharbi, S. M., Bazid, M. A. A. Gendy Mahmoud S. E., 2010, "Heat and Mass Transfer in MHD Visco-Elastic Fluid Flow through a Porous Medium over a Stretching Sheet with Chemical Reaction," Applied Mathematics, 1, 446-455,

http://dx.doi.org/10.4236/am.2010.16059

Barik, R. N. and Dash, G. C., 2014 "Thermal Radiation Effect on an Unsteady Magnetohydrodynamic Flow Past Inclined Porous Heated Plate in the Presence of Chemical Reaction and Viscous Dissipation", Applied Mathematics and Computation, 226, 423-434.

https://doi.org/10.1016/j.amc.2013.09.077

Bhavana, M., Kesavaiah, D.C., Sudhakaraiah, A., 2013 "The Soret Effect on Free Convective Unsteady MHD Flow over a Vertical Plate with Heat Source," International Journal of Innovative Research in Science, Engineering and Technology, 2(5), ISSN: 2319-8753.

Bhuvaneswari, M., Sivasankaran, S. and Kim, Y. J., 2010 "Exact Analysis of Radiation Convective Flow Heat and Mass Transfer over an Inclined Plate in a Porous Medium", World Applied Sciences Journal, 10 (7), 774-778.

Chandra Reddy, P., Raju, M. C. and Raju, G. S. S.,2016, "Soret and Dufour Effects on MHD Eree Convection Flow of Rivlin-Ericksen Fluid Past a Semi-infinite Vertical Plate," Advances and Applications in Fluid Mechanics, 19 (2), 401-414.

Ibrahim, S. M., 2014, "Effects of Chemical Reaction on Dissipative Radiative MHD Flow through a Porous Medium over a Non-isothermal Stretching Sheet," Hindawi Publishing Corporation Journal of Industrial Mathematics, 2014, Article ID 243148, 1-10. http://dx.doi.org/10.1155/2014/243148

Jena S., Dash, G.C., Mishra, S. R., 2016, "Chemical Reaction Effect on MHD Viscoelastic Fluid Flow over a Vertical Stretching Sheet with Heat Source/Sink," Ain Shams Engineering Journal,1-9.

http://dx.doi.org/10.1016/j.asej.2016.06.014

Jonnadula, M., Polarapu, P., Reddy, M.G., Venakateswarlu, M., 2015, "Influence of Thermal Radiation and Chemical Reaction on MHD Flow, Heat and Mass Transfer over a Stretching Surface," International Conference on Computational Heat and Mass Transfer-2015 Science Direct Procedia Engineering, 127, 1315 - 1322.

http://doi.org/10.1016/j.proeng.2015.11.489

Mamatha, B., Raju, M. C., and Varma S. V. K., 2015, "Thermal Diffusion Effect on MHD Mixed Convection Unsteady Flow of a Micro Polar Fluid Past a Semi-Infinite Vertical Porous Plate with Radiation and Mass Transfer," International Journal of Engineering Research in Africa, 13, 21-37.

http://dx.doi.org/10.4028/www.scientific.net/JERA.13.21

Manglesh, A. and Gorla, M.G., 2012, "The Effects of Thermal Radiation, Chemical Reaction and Rotation on Unsteady MHD Viscoelastic Slip Flow," Global Journal of Science Frontier Research Mathematics and Decision Sciences, 12 (14), Version 1.0. 1-15.

Metri, P.G., Metri, P. G., Abel, S., Silvestrov, S., 2016, "Heat Transfer in MHD Mixed Convection Viscoelastic Fluid Flow over a Stretching Sheet Embedded in a Porous Medium with Viscous Dissipation and
Non-uniform Heat Source/Sink," IX International Conference on Computational Heat and Mass Transfer, ICCHMT.

http://doi.org/10.1016/j.proeng.2016.08.371

Misra, J.C. and Adhikary, S.D., 2016, "MHD Oscillatory Channel Flow, Heat and Mass Transfer in a Physiological Fluid in Presence of Chemical Reaction," Alexandria Engineering Journal, 55, 287-297. http://dx.doi.org/10.1016/j.aej.2015.10.005

Mondal, H., Pal, D., Chatterjee, S., and Sibanda P., 2017, "Thermophoresis and Soret-Dufour on MHD Mixed Convection Mass Transfer over an Inclined Plate with Non-uniform Heat Source/Sink and Chemical Reaction," Ain Shams Engineering Journal, https://doi.org/ 10.1016/ j.asej.2016.10.015

Nayak, M.K., Dash, G.C., Singh, L.P. 2016, "Heat and Mass Transfer Effects on MHD Viscoelastic Fluid over a Stretching Sheet Through Porous Medium Inpresence of Chemical Reaction, Propulsion and Power Research, 5(1),70-80.

http://dx.doi.org/10.1016/j.j ppr.2016.01.006

Pal, D., Mondal, H., 2012, "Influence of Chemical Reaction and Thermal Radiation on Mixed Convection Heat and Mass Transfer over a Stretching Sheet in Darcian Porous Medium with Soret and Dufour Effects," 62, 102-108

https://doi.org/10.1016/j. enconman.2012.03.017.

Pal, D., Talukdar, B., 2011, "Combined Effects of Joule Heating and Chemical Reaction on Unsteady Magnetohydrodynamic Mixed Convection of a Viscous Dissipating Fluid over a Vertical Plate in Porous Media with Thermal Radiation," Mathematical and Computer Modelling, 54, 3016-3036.

Raju, M.C., Veeresh, C., Varma, S.V.K., Kumar, B. R. and Kumar A.G.V., 2015, "Heat and Mass Transfer in MHD Mixed Convection Flow on a Moving Inclined Porous Plate, "Journal of Applied and Computed Math, 4(5)

http://dx.doi.org/10.4172/2168-9679.1000259

Ramana Reddy, J.V., Sugunamma, V., Sandeep, N., Sulochana, C., 2016, "Influence of Chemical Reaction, Radiation and Rotation on MHD Nano Fluid Flow Past a Permeable Flat Plate in Porous Medium," Journal of the Nigerian Mathematical Society 35 (2016) 4865.

http://dx.doi.org/10.1016/j.jnnms.2015.08.004

Ramandevi, B., Ramana Reddy, J.V., Sugunamma, V., Sandeep, N., 2017, "Combined Influence of Viscous Dissipation and non Uniform Heat Source/Sink on MHD non-Newtonian Fluid Flow with CattaneoChristov Heat Flux," Alexandria Engineering Journal. http://dx.doi.org/10.1016/j.aej. 2017.01.026

Rashidi, M.M., Ali, M., Rostami, R., Rostami, P., and Xie G.N., 2015, "Heat and Mass Transfer for MHD Viscoelastic Fluid Flow over a Vertical Stretching Sheet with Considering Soret and Dufour Effects," Hindawi Publishing Corporation Mathematical Problems in Engineering, 2015, Article ID 861065, 12 pages.

http://dx.doi.org/10.1155/2015/861065

Ravikumar,V., Raju, M.C. and Raju, G.S.S.,2012, "Heat and Mass Transfer Effects on MHD Flow of Viscous Fluid Through NonHomogeneous Porous Medium in Presence of Temperature Dependent Heat Source," International Journal of Contemporary Mathematical Sciences, 7(32), 1597 - 1604. 
Reddy, C. P., Raju, M. C., and Raju, G. S. S., 2016, "Soret and Dufour Effects on MHD Free Convection Flow of Rivlin-Ericksen Fluid Past a Semi-Infinite Vertical Plate," Advances and Applications in Fluid Mechanics. 19(2), 401-414.

http://dx.doi.org/10.17654/FM019020401

Reddy, M.G., Padma, P., Shankar, B., 2015, "Effects of Viscous Dissipation and Heat Source on Unsteady MHD Flow over a Stretching Sheet," Ain Shams Engineering Journal, 6,1195-1201

http://dx.doi.org/10.1016/j.asej.2015.04.006

Rudraswamy, N. G. and Gireesha, J., 2014, "Influence of Chemical Reaction and Thermal Radiation on MHD Boundary Layer Flow and Heat Transfer of a Nano fluid over an Exponentially Stretching Sheet," Journal of Applied Mathematics and Physics, 2, 24-32 http://dx.doi.org/10.4236/jamp.2014.22004

Sengupta, S. and Ahmed, N., 2015, "Chemical Reaction Interaction on Unsteady MHD free Convective Radiative Flow Past an Oscillating Plate Embedded in Porous Media with Thermal Diffusion," Advances in Applied Science Research, 6(7), 87-104.

Sengupta, S. and Karmakar, A., 2016, "MHD Mixed Convection Chemically Reactive Flow in Radiative Heat Generating Medium with Soret Effect," International Journal of Modern Mathematical Sciences. 14(3), 239-261.

Sengupta, S. and Karmakar, A., 2018, "Induced Magnetic Field Interaction in Free Convective Heat and Mass Transfer Flow of a Chemically Reactive Heat Generating Fluid with Thermo-diffusion and Diffusion-thermo Effects," Frontiers in Heat and Mass Transfer (FHMT), 10(19).

http://dx.doi.org/10.5098/hmt.10.19

Sengupta, S. and Sen, M., 2013, "Free Convective Heat and Mass Transfer Flow Past an Oscillating Plate with Heat Generation, Thermal Radiation and Thermo Diffusion Effects," J.P J. of heat and mass transfer, 8(2), 187-210.

Sengupta, S., 2011, "Thermal Diffusion Effect of Eree Convection Mass Transfer Flow Past a Uniformly Accelerated Porous Plate with Heat Sink," International Journal of Mathematical Archive, 2(8), 12661273.

Sengupta, S., 2014, "Heat and Mass Transfer Phenomena in MHD Flow Modelling with Applications" Scientific Research Publishing, Incorporated, ISBN 1618960660, 9781618960665.

Sengupta, S., 2015, “An Analysis on Unsteady Heat and Mass Transfer Flow of Radiative Chemically Reactive Fluid past an Oscillating Plate Embedded In Porous Media in Presence of Soret Effect," International Journal of Recent Technology and Engineering, 3(6), 10-14.

Sengupta, S., 2015, "Free Convective Chemically Absorption Fluid Past an Impulsively Accelerated Plate with Thermal Radiation Variable Wall Temperature and Concentrations," Applications and Applied Mathematics, 10(1), $328-348$.

Sengupta, S., and Ahmed, N., 2016, "MHD Free Convective Mass Transfer Flow of Radiative Uniform Heat Generation (Absorption) Fluid through a Wavy Permeable Channel in Presence of Soret and Dufour Effects," Canadian Journal of Physics, 95 (1), 44-58. https://doi.org /10.1139/cjp - 2014-0599

Shamshuddin, M.D., 2017, "Heat and Mass Transfer on the Unsteady MHD Flow of Chemically Reacting Micropolar Fluid with Radiation and Joule Heating,"International Journal of Theoretical and Applied Mathematics, 3(3), 110-121.

DOI: $10.11648 /$ j.ijtam.20170303.13

Shamshuddin, M.D., Chamkha, A.J., Thumma T, Raju M.C., 2018, "Computation of Unsteady MHD Mixed Convective Heat and Mass Transfer in Dissipative Reactive Micropolar Flow considering Soret and Dufour Effects," Frontiers in Heat and Mass Transfer, 10, pp. 115,2018

http://dx.doi.org/10.5098/hmt.10.15

Srinivasacharya, D. and Swamy Reddy, G.,2016, "Chemical reaction and radiation effects on mixed convection heat and mass transfer over a vertical plate in power-law fluid saturated porous medium," Journal of the Egyptian Mathematical Society, 24, 108-115

http://dx.doi.org /10.1016/j.joems.2014.10.001

Uddin M.N., Alim, M.A., Chowdhury, M.M.K.,2014, "Effect of Conjugate Heat and Mass Transfer on MHD Mixed Convective Flow past Inclined Porous Plate in Porous Medium," International Journal of Mechanical, Aerospace, Industrial and Mechatronics Engineering, $8(3), 825-829$.

Ullah, I., Khan, I., Shafie, S., 2017, "Soret and Dufour Effects on Unsteady Mixed Convection Slip Flow of Casson Fluid over a Nonlinearly Stretching Sheet with Convective Boundary Condition," Scientific Reports, 7(1113).

http://dx.doi.org/10.1038/s41598-017-01205-5

Venkataramana, B. S., Jalaja, P., and Jayakumar, R. K., 2017, "The Influence of Heat Generation (Absorption) and Thermal Radiation on MHD Laminar Boundary Layer Flow over a Moving Cylindrical Rod," International Journal of engineering Sciences and Research Technology. 6(2), 471-475.

http://dx.doi.org/10.5281/zenodo.293743

\section{APPENDIX}

$$
\begin{aligned}
& x_{1}=\frac{\left[\operatorname{Pr}+\frac{1}{\sqrt{3}} \sqrt{3 \operatorname{Pr}^{2}-4(3+4 R) Q s \operatorname{Pr}}\right]}{2\left(1+\frac{4 R}{3}\right)} x_{2}=\frac{\left[S c+\sqrt{S c^{2}+4 C_{R} S c}\right]}{2} \\
& x_{3}=\frac{1}{2}\left[1+\sqrt{1+4 M_{1}}\right] \quad x_{4}=x_{1} \quad x_{5}=x_{2} \quad x_{6}=x_{3} \quad x_{7}=0 \\
& x_{8}=b_{1}+i b_{2} \quad x_{9}=\mathrm{b}_{3}+i b_{4} \\
& x_{10}=\mathrm{b}_{5}+i b_{6}, \quad x_{11}=\mathrm{b}_{7}+i b_{8}, \quad x_{12}=\mathrm{b}_{9}+i b_{10} \\
& b_{1}=\frac{\lambda S c}{2}+\frac{1}{2 \sqrt{2}}\left[\sqrt{\left(S c^{2} \lambda^{2}+4 C_{R} S c\right)^{2}+16 \omega^{2} S c^{2}}+\left(S c^{2} \lambda^{2}+4 C_{R} S c\right)\right]^{\frac{1}{2}} \\
& b_{2}=\frac{1}{2 \sqrt{2}}\left[\sqrt{\left(S c^{2} \lambda^{2}+4 C_{R} S c\right)^{2}+16 \omega^{2} S c^{2}}-\left(S c^{2} \lambda^{2}+4 C_{R} S c\right)\right]^{\frac{1}{2}} \\
& b_{3}=\frac{\lambda}{2}+\frac{1}{2 \sqrt{2}}\left[\sqrt{\left(\lambda^{2}+4 M_{1}\right)^{2}}+16 \omega^{2}+\left(\lambda^{2}+4 M_{1}\right)\right]^{\frac{1}{2}} \\
& b_{4}=\frac{1}{2 \sqrt{2}}\left[\sqrt{\left(\lambda^{2}+4 M_{1}\right)^{2}}+16 \omega^{2}-\left(\lambda^{2}+4 M_{1}\right)\right]^{\frac{1}{2}} \\
& b_{5}=\frac{\lambda \operatorname{Pr}}{2\left(1+\frac{4 R}{3}\right)}+\frac{1}{2 \sqrt{2}\left(1+\frac{4 R}{3}\right)}\left[\begin{array}{l}
\sqrt{\left(\operatorname{Pr}^{2} \lambda-4\left(1+\frac{4 R}{3}\right) Q s \operatorname{Pr}\right)^{2}+16\left(1+\frac{4 R}{3}\right)^{2} \operatorname{Pr}^{2} \omega^{2}} \\
+\left(\operatorname{Pr}^{2} \lambda-4\left(1+\frac{4 R}{3}\right) Q s \operatorname{Pr}\right)
\end{array}\right]
\end{aligned}
$$




$$
\begin{aligned}
& b_{6}=\frac{1}{2 \sqrt{2}\left(1+\frac{4 R}{3}\right)}\left[\begin{array}{l}
\sqrt{\left(\operatorname{Pr}^{2} \lambda-4\left(1+\frac{4 R}{3}\right) Q s \operatorname{Pr}\right)^{2}+16\left(1+\frac{4 R}{3}\right)^{2} \operatorname{Pr}^{2} \omega^{2}} \\
-\left(\operatorname{Pr}^{2} \lambda-4\left(1+\frac{4 R}{3}\right) Q s \operatorname{Pr}\right)
\end{array}\right]^{\frac{1}{2}} \\
& b_{7}=b_{1}, \quad b_{8}=b_{2}, \quad b_{9}=b_{3}, \quad b_{10}=b_{4} \\
& \mathrm{~A}_{1}=\frac{-G m}{x_{2}^{2}-x_{2}-M_{1}} \quad \mathrm{~A}_{2}=\frac{-G m d_{2}}{x_{2}^{2}-x_{2}-M_{1}} \quad \mathrm{~A}_{3}=\frac{-G m d_{1}}{x_{1}^{2}-x_{1}-M_{1}} \\
& \mathrm{~A}_{4}=\mathrm{A}_{1}+\mathrm{A}_{2} \quad \mathrm{~A}_{5}=\mathrm{u}_{p}-\left(1+d_{2}+\mathrm{A}_{4}+\mathrm{A}_{3}\right) \quad \mathrm{A}_{6}=d_{2}+A_{3} \\
& \mathrm{~A}_{7}=\frac{-\operatorname{Pr} x_{3}^{2} A_{5}^{2}}{\left(1+\frac{4 R}{3}\right)\left(2 x_{3}\right)^{2}-2 \operatorname{Pr} x_{3}+Q s \operatorname{Pr}} \\
& \mathrm{A}_{8}=\frac{-\operatorname{Pr} x_{2}^{2} A_{4}^{2}}{\left(1+\frac{4 R}{3}\right)\left(2 x_{2}\right)^{2}-2 \operatorname{Pr} x_{2}+Q s \operatorname{Pr}} \\
& \mathrm{A}_{9}=\frac{-\operatorname{Pr} x_{1}^{2} A_{6}^{2}}{\left(1+\frac{4 R}{3}\right)\left(2 x_{1}\right)^{2}-2 \operatorname{Pr} x_{1}+Q s \operatorname{Pr}} \\
& \mathrm{A}_{10}=\frac{-\operatorname{Pr} x_{3} x_{2} A_{4} A_{5}}{\left(1+\frac{4 R}{3}\right)\left(x_{3}+x_{2}\right)^{2}-\operatorname{Pr}\left(x_{3}+x_{2}\right)+Q s \operatorname{Pr}} \\
& \mathrm{A}_{11}=\frac{-\operatorname{Pr} x_{1} x_{2} A_{4} A_{6}}{\left(1+\frac{4 R}{3}\right)\left(x_{1}+x_{2}\right)^{2}-\operatorname{Pr}\left(x_{1}+x_{2}\right)+Q s \operatorname{Pr}} \\
& \mathrm{A}_{12}=\frac{-\operatorname{Pr} x_{3} x_{1} A_{6} A_{5}}{\left(1+\frac{4 R}{3}\right)\left(x_{3}+x_{1}\right)^{2}-\operatorname{Pr}\left(x_{3}+x_{1}\right)+Q s \operatorname{Pr}} \text {, } \\
& \mathrm{A}_{13}=\frac{-S r S c x_{4}^{2} d_{3}}{x_{4}^{2}-S c x_{4}-C_{R} S c} \mathrm{~A}_{14}=\frac{-S r S c\left(2 x_{3}\right)^{2} A_{7}}{\left(2 x_{3}\right)^{2}-2 S c x_{3}-C_{R} S c} \\
& \mathrm{~A}_{15}=\frac{-S r S c\left(2 x_{2}\right)^{2} A_{8}}{\left(2 x_{2}\right)^{2}-2 S c x_{2}-C_{R} S c} \quad \mathrm{~A}_{16}=\frac{-S r S c\left(2 x_{1}\right)^{2} A_{9}}{\left(2 x_{1}\right)^{2}-2 S c x_{1}-C_{R} S c} \\
& \mathrm{~A}_{17}=\frac{-S r S c A_{10}}{\left(x_{3}+x_{2}\right)^{2}-S c\left(x_{3}+x_{2}\right)-C_{R} S c} \text {, } \\
& \mathrm{A}_{18}=\frac{-S r S c A_{11}}{\left(x_{1}+x_{2}\right)^{2}-S c\left(x_{1}+x_{2}\right)-C_{R} S c} \\
& \mathrm{~A}_{19}=\frac{-S r S c A_{12}}{\left(x_{1}+x_{3}\right)^{2}-S c\left(x_{1}+x_{3}\right)-C_{R} S c} \\
& \mathrm{~A}_{20}=\frac{-G r d_{3}}{x_{4}^{2}-x_{4}-M_{1}} \quad \mathrm{~A}_{21}=\frac{-G r A_{7}}{\left(2 x_{3}\right)^{2}-2 x_{3}-M_{1}} \\
& \mathrm{~A}_{22}=\frac{-G r A_{8}}{\left(2 x_{2}\right)^{2}-2 x_{2}-M_{1}} \quad \mathrm{~A}_{23}=\frac{-G r A_{9}}{\left(2 x_{1}\right)^{2}-2 x_{1}-M_{1}} \text {, } \\
& \mathrm{A}_{24}=\frac{-G r A_{10}}{\left(x_{3}+x_{2}\right)^{2}-\left(x_{3}+x_{2}\right)-M_{1}}
\end{aligned}
$$

$$
\begin{aligned}
& \mathrm{A}_{25}=\frac{-G r A_{11}}{\left(x_{1}+x_{2}\right)^{2}-\left(x_{1}+x_{2}\right)-M_{1}} \quad \mathrm{~A}_{26}=\frac{-G r A_{12}}{\left(x_{3}+x_{1}\right)^{2}-\left(x_{3}+x_{1}\right)-M_{1}} \\
& \mathrm{~A}_{27}=\frac{-G r d_{4}}{x_{5}^{2}-x_{5}-M_{1}} \mathrm{~A}_{28}=\frac{-G m A_{13}}{x_{4}^{2}-x_{4}-M_{1}} \quad \mathrm{~A}_{29}=\frac{-G m A_{14}}{\left(2 x_{3}\right)^{2}-2 x_{3}-M_{1}} \\
& \mathrm{~A}_{30}=\frac{G m A_{15}}{\left(2 x_{2}\right)^{2}-2 x_{2}-M_{1}} \quad \mathrm{~A}_{31}=\frac{-G m A_{16}}{\left(2 x_{3}\right)^{2}-2 x_{3}-M_{1}} \\
& \mathrm{~A}_{32}=\frac{G m A_{17}}{\left(x_{3}+x_{2}\right)^{2}-\left(x_{3}+x_{2}\right)-M_{1}} \mathrm{~A}_{33}=\frac{G m A_{18}}{\left(x_{1}+x_{2}\right)^{2}-\left(x_{1}+x_{2}\right)-M_{1}} \\
& A_{35}=A_{20}+A_{28}, A_{36}=A_{21}+A_{29} \text {, }
\end{aligned}
$$

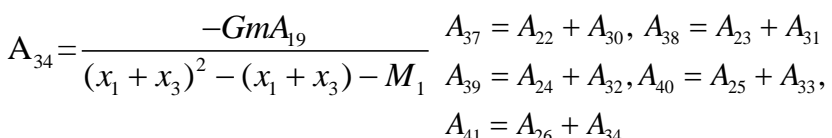

$$
\begin{aligned}
& \mathrm{A}_{42}=\frac{d_{10}}{d_{10}^{2}+d_{11}^{2}} \quad \mathrm{~A}_{43}=\frac{d_{11}}{d_{10}^{2}+d_{11}^{2}} \\
& A_{44}=A_{42} b_{3}+A_{43} b_{4}, \quad A_{45}=A_{42} b_{4}-A_{43} b_{3} \quad \mathrm{~A}_{46}=\frac{A_{44} d_{15}+A_{45} d_{16}}{d_{15}^{2}+d_{16}^{2}} \\
& \mathrm{~A}_{47}=\frac{A_{45} d_{15}-A_{44} d_{16}}{d_{15}^{2}+d_{16}^{2}} \quad \mathrm{~A}_{48}=\frac{A_{44} d_{17}+A_{45} d_{18}}{d_{17}^{2}+d_{18}^{2}} \mathrm{~A}_{49}=\frac{A_{45} d_{17}-A_{44} d_{18}}{d_{17}^{2}+d_{18}^{2}} \\
& \mathrm{~A}_{50}=\frac{A_{44} d_{19}+A_{45} d_{20}}{d_{19}^{2}+d_{20}^{2}} \quad \mathrm{~A}_{51}=\frac{A_{45} d_{19}-A_{44} d_{20}}{d_{19}^{2}+d_{20}^{2}} \\
& A_{52}=A_{42} b_{1}+A_{43} b_{2}, \quad A_{53}=A_{42} b_{2}-A_{43} b_{1} \quad \mathrm{~A}_{54}=\frac{A_{53} d_{21}-A_{53} d_{22}}{d_{21}^{2}+d_{22}^{2}} \\
& \mathrm{~A}_{55}=\frac{A_{53} d_{21}-A_{52} d_{22}}{d_{21}^{2}+d_{22}^{2}} \mathrm{~A}_{56}=\frac{A_{52} d_{23}-A_{53} d_{24}}{d_{23}^{2}+d_{24}^{2}} \mathrm{~A}_{57}=\frac{A_{53} d_{23}-A_{52} d_{24}}{d_{23}^{2}+d_{24}^{2}} \\
& \mathrm{~A}_{58}=\frac{A_{52} d_{25}+A_{53} d_{26}}{d_{25}^{2}+d_{26}^{2}} \mathrm{~A}_{59}=\frac{A_{53} d_{25}-A_{52} d_{26}}{d_{25}^{2}+d_{26}^{2}} \mathrm{~A}_{60}=\frac{x_{3} A_{5}\left(a_{10} d_{15}+a_{11} d_{16}\right)}{d_{15}^{2}+d_{16}^{2}} \\
& \mathrm{~A}_{61}=\frac{x_{3} A_{5}\left(a_{11} d_{15}-a_{10} d_{16}\right)}{d_{15}^{2}+d_{16}^{2}} \mathrm{~A}_{62}=\frac{x_{2} A_{4}\left(b_{3} d_{17}+b_{4} d_{18}\right)}{d_{17}^{2}+d_{18}^{2}} \\
& \mathrm{~A}_{63}=\frac{x_{2} A_{4}\left(b_{4} d_{17}-b_{3} d_{18}\right)}{d_{17}^{2}+d_{18}^{2}} \mathrm{~A}_{64}=\frac{x_{1} A_{6}\left(b_{3} d_{19}+b_{4} d_{20}\right)}{d_{19}^{2}+d_{20}^{2}} \\
& \mathrm{~A}_{65}=\frac{x_{1} A_{6}\left(b_{4} d_{19}-b_{3} d_{20}\right)}{d_{19}^{2}+d_{20}^{2}} A_{66}=d_{43}+d_{45}+d_{47}-d_{37}-d_{39}-d_{41} \\
& A_{67}=d_{44}+d_{46}+d_{48}-d_{38}-d_{40}-d_{42} \\
& \mathrm{~A}_{68}=\frac{A_{66} b_{5}+A_{67} b_{6}}{b_{5}^{2}+b_{6}^{2}} \quad \mathrm{~A}_{69}=\frac{A_{67} b_{5}-A_{66} b_{6}}{b_{5}^{2}+b_{6}^{2}} \quad \mathrm{~A}_{70}=\frac{d_{67} d_{81}+d_{68} d_{82}}{d_{81}^{2}+d_{82}^{2}} \\
& \mathrm{~A}_{71}=\frac{d_{68} d_{81}-d_{67} d_{82}}{d_{81}^{2}+d_{82}^{2}} \quad \mathrm{~A}_{72}=\frac{d_{69} d_{83}+d_{70} d_{84}}{d_{83}^{2}+d_{84}^{2}} \quad \mathrm{~A}_{73}=\frac{d_{70} d_{83}+d_{69} d_{87}}{d_{83}^{2}+d_{84}^{2}} \\
& \mathrm{~A}_{74}=\frac{d_{71} d_{85}+d_{72} d_{86}}{d_{85}^{2}+d_{86}^{2}} \mathrm{~A}_{75}=\frac{d_{72} d_{85}-d_{71} d_{86}}{d_{86}^{2}+d_{86}^{2}} \\
& \mathrm{~A}_{76}=\frac{d_{73} d_{87}-d_{74} d_{88}}{d_{87}^{2}+d_{88}^{2}} \quad \mathrm{~A}_{77}=\frac{d_{74} d_{87}-d_{73} d_{88}}{d_{87}^{2}+d_{88}^{2}} \quad \mathrm{~A}_{78}=\frac{d_{75} d_{89}+d_{76} d_{90}}{d_{89}^{2}+d_{90}^{2}} \\
& \mathrm{~A}_{79}=\frac{d_{76} d_{89}-d_{75} d_{90}}{d_{89}^{2}+d_{90}^{2}} \quad \mathrm{~A}_{80}=\frac{d_{77} d_{91}+d_{78} d_{92}}{d_{91}^{2}+d_{92}^{2}} \quad \mathrm{~A}_{81}=\frac{d_{78} d_{91}-d_{77} d_{92}}{d_{91}^{2}+d_{92}^{2}} \\
& \mathrm{~A}_{82}=\frac{d_{79} d_{93}+d_{80} d_{94}}{d_{93}^{2}+d_{94}^{2}} \quad \mathrm{~A}_{83}=\frac{d_{80} d_{93}-d_{79} d_{94}}{d_{93}^{2}+d_{94}^{2}} \\
& \mathrm{~A}_{84}=\frac{G m S c S r\left(d_{95} d_{111}+d_{96} d_{112}\right)}{d_{111}^{2}+d_{112}^{2}} \mathrm{~A}_{85}=\frac{\operatorname{GmScSr}\left(d_{96} d_{111}-d_{95} d_{112}\right)}{d_{111}^{2}+d_{112}^{2}}
\end{aligned}
$$




$$
\begin{aligned}
& \mathrm{A}_{86}=\frac{d_{97} d_{113}+d_{98} d_{114}}{d_{113}^{2}+d_{114}^{2}} \mathrm{~A}_{87}=\frac{d_{98} d_{113}-d_{97} d_{114}}{d_{113}^{2}+d_{114}^{2}} \\
& \mathrm{~A}_{88}=\frac{d_{99} d_{115}-d_{100} d_{116}}{d_{115}^{2}+d_{116}^{2}} \mathrm{~A}_{89}=\frac{d_{100} d_{115}-d_{99} d_{116}}{d_{115}^{2}+d_{116}^{2}} \\
& \mathrm{~A}_{90}=\frac{d_{101} d_{117}+d_{102} d_{118}}{d_{117}^{2}+d_{118}^{2}} \quad \mathrm{~A}_{91}=\frac{d_{102} d_{117}-d_{101} d_{118}}{d_{117}^{2}+d_{118}^{2}} \\
& \mathrm{~A}_{92}=\frac{d_{103} d_{119}+d_{104} d_{120}}{d_{119}^{2}+d_{120}^{2}} \quad \mathrm{~A}_{93}=\frac{d_{104} d_{119}-d_{103} d_{120}}{d_{119}^{2}+d_{120}^{2}} \\
& \mathrm{~A}_{94}=\frac{d_{105} d_{121}+d_{106} d_{122}}{d_{121}^{2}+d_{122}^{2}} \quad \mathrm{~A}_{95}=\frac{d_{106} d_{121}-d_{105} d_{122}}{d_{121}^{2}+d_{122}^{2}} \\
& \mathrm{~A}_{96}=\frac{d_{107} d_{123}+d_{108} d_{124}}{d_{123}^{2}+d_{124}^{2}} \mathrm{~A}_{97}=\frac{d_{108} d_{123}-d_{107} d_{124}}{d_{123}^{2}+d_{124}^{2}} \\
& \mathrm{~A}_{98}=\frac{d_{109} d_{125}+d_{110} d_{126}}{d_{125}^{2}+d_{126}^{2}} \quad \mathrm{~A}_{99}=\frac{d_{110} d_{125}-d_{109} d_{126}}{d_{125}^{2}+d_{126}^{2}} \\
& A_{100}=A_{84}+A_{86}+A_{88}+A_{90}+A_{92}+A_{94}+A_{96}+A_{98} \\
& A_{101}=A_{85}+A_{87}+A_{89}+A_{91}+A_{93}+A_{95}+A_{97}+A_{99} \\
& d_{1}=\frac{S c S r x_{1}}{x_{1}^{2}-S c x_{1}-C_{R} S c} \quad d_{2}=\frac{G r}{x_{1}\left(x_{1}^{2}-x_{1}-M_{1}\right)} \\
& d_{3}=-\frac{1}{x_{4}}\left[\begin{array}{l}
2 x_{3} A_{7}+2 x_{2} A_{8}+2 x_{1} A_{9}+\left(x_{2}+x_{3}\right) A_{10} \\
+\left(x_{2}+x_{1}\right) A_{11}+\left(x_{1}+x_{3}\right) A_{12}
\end{array}\right] \\
& d_{4}=-\left(A_{13}+A_{14}+A_{15}+A_{16}+A_{17}+A_{18}+A_{19}\right) \\
& d_{5}=-\left(d_{6}+d_{7}+d_{8}+d_{9}\right) \\
& d_{6}=A_{20}+A_{21}, d_{7}=A_{22}+A_{23}, d_{8}=A_{24}+A_{25}+\mathrm{A}_{26} \\
& d_{9}=A_{27}+A_{28}+A_{29}+A_{30}+A_{31}+A_{32}+A_{33}+A_{34} \\
& d_{10}=b_{1}^{2}-b_{2}^{2}-\lambda b_{1}-M_{1}, \quad d_{11}=2 b_{1} b_{2}-\lambda b_{2}-\omega \\
& d_{12}=x_{3}^{2}+b_{3}^{2}-b_{4}^{2}+2 x_{3} b_{3}, \quad d_{13}=\lambda \operatorname{Pr}\left(x_{3}+b_{3}\right)+Q s \operatorname{Pr} \\
& d_{14}=1+\frac{4 R}{3} \quad, \quad d_{15}=d_{14} d_{12}-d_{13} \\
& d_{16}=d_{14}\left(2 b_{3} b_{4}+2 x_{3} b_{4}\right)-\lambda \operatorname{Pr} b_{4}-\omega \operatorname{Pr} \\
& d_{17}=d_{14}\left(x_{2}^{2}+b_{3}^{2}-b_{4}^{2}+2 x_{2} b_{3}\right)-\lambda \operatorname{Pr}\left(x_{2}+b_{3}\right)+Q s \operatorname{Pr} \\
& d_{18}=d_{14}\left(2 b_{3} b_{4}+2 x_{2} b_{4}\right)-\lambda \operatorname{Pr} b_{4}-\omega \operatorname{Pr} \text {, } \\
& d_{19}=d_{14}\left(x_{1}^{2}+b_{3}^{2}-b_{4}^{2}+2 x_{1} b_{3}\right)-\lambda \operatorname{Pr}\left(x_{1}+b_{3}\right)+Q s \operatorname{Pr} \\
& d_{20}=d_{14}\left(2 b_{3} b_{4}+2 x_{1} b_{4}\right)-\lambda \operatorname{Pr} b_{4}-\omega \operatorname{Pr} \\
& d_{21}=d_{14}\left(x_{3}^{2}+b_{1}^{2}-b_{2}^{2}+2 x_{3} b_{1}\right)-\lambda \operatorname{Pr}\left(x_{3}+b_{1}\right)+Q s \operatorname{Pr} \\
& d_{22}=d_{14}\left(2 b_{1} b_{2}+2 x_{3} b_{2}\right)-\lambda \operatorname{Pr} b_{2}-\omega \operatorname{Pr} \\
& d_{23}=d_{14}\left(x_{2}^{2}+b_{1}^{2}-b_{2}^{2}+2 x_{2} b_{1}-\lambda \operatorname{Pr}\left(x_{2}+b_{1}\right)+Q s \operatorname{Pr}\right. \\
& d_{24}=d_{14}\left(2 b_{1} b_{2}+2 x_{2} b_{2}\right)-\lambda \operatorname{Pr} b_{2}-\omega \operatorname{Pr} \\
& d_{25}=d_{14}\left(x_{1}^{2}+b_{1}^{2}-b_{2}^{2}+2 x_{1} b_{1}-\lambda \operatorname{Pr}\left(x_{1}+b_{1}\right)+Q s \operatorname{Pr}\right. \\
& d_{26}=d_{14}\left(2 b_{1} b_{2}+2 x_{1} b_{2}\right)-\lambda \operatorname{Pr} b_{2}-\omega \operatorname{Pr} \\
& d_{27}=G m \cos \psi \quad d_{28}=x_{3} A_{5} \quad d_{29}=x_{2} A_{4} \quad d_{30}=x_{1} A_{6} \\
& d_{31}=d_{27} d_{28} A_{46}-A_{60} \quad d_{32}=d_{27} d_{28} A_{47}-A_{61} \\
& d_{33}=d_{27} d_{29} A_{48}-A_{62} \quad d_{34}=d_{27} d_{29} A_{49}-A_{63} \\
& d_{35}=d_{27} d_{30} A_{50}-A_{64} \quad d_{36}=d_{27} d_{30} A_{51}-A_{65} \\
& d_{37}=d_{27} d_{28}\left(A_{54}\left(x_{3}+b_{1}\right)-A_{55} b_{2}\right) d_{38}=d_{27} d_{28}\left(A_{55}\left(x_{3}+b_{1}\right)+A_{54} b_{2}\right) \\
& d_{39}=d_{27} d_{29}\left(A_{56}\left(x_{2}+b_{1}\right)-A_{57} b_{2}\right) d_{40}=d_{27} d_{29}\left(A_{57}\left(x_{2}+b_{1}\right)+A_{56} b_{2}\right) \\
& d_{41}=d_{27} d_{30}\left(A_{58}\left(x_{1}+b_{1}\right)-A_{59} b_{2}\right) d_{42}=d_{27} d_{30}\left(A_{59}\left(x_{1}+b_{1}\right)+A_{58} b_{2}\right) \\
& \left.d_{43}=d_{31}\left(x_{3}+b_{3}\right)-d_{32} b_{4}\right) d_{44}=d_{32}\left(x_{3}+b_{3}\right)+d_{31} b_{4}
\end{aligned}
$$

